\title{
Invariance of the psychometric function for character recognition across the visual field
}

\author{
HANS STRASBURGER \\ Ludwig-Maximilians-Universität, München, Germany \\ and Otto-von-Guericke-Universität, Magdeburg, Germany
}

\begin{abstract}
The psychometric function for recognition of singly presented digits as a function of digit contrast was measured at $2^{\circ}$ steps across the horizontal meridian of the visual field, under monocular and binocular viewing conditions. A maximum-likelihood staircase procedure was used in a 10-alternative forcedchoice recognition paradigm to gather the data. Both the Weibull and the logistic psychometric functions provide excellent fits to the observed data. The slopes of these functions at their point of inflection ranged from 4.0 to 5.0 proportion-correct $/ \log _{10}$-unit contrast, for both monocular and binocular viewing and for all loci in the visual field. These slope values correspond to short-term measurements (around 30 trials, or $1 \mathrm{~min}$ ) and do not include performance variations of longer duration; the latter are estimated to increase slope by a factor of about 1.5. A single psychometric function shape, centered around a threshold value, therefore describes recognition performance at all retinal loci and binocularity. An empirical comparison of slope results across the literature shows that the function's slope is about twice that reported for a number of detection tasks. The comparison of recognition contrast thresholds, percentage correct values, and other performance measures across studies requires the knowledge of the psychometric function's slope, and our results thus provide a firm basis for the study of low-contrast character recognition.
\end{abstract}

Low-contrast letter recognition has been studied since the previous century (Berry, 1889) but has gained increased interest in the past years, both in basic research (Alexander, Xie, \& Derlacki, 1993, 1997; Higgins, Arditi, \& Knoblauch, 1996; Legge, Rubin, \& Luebker, 1987; Solomon \& Pelli, 1994; Strasburger, Harvey, \& Rentschler, 1991; Strasburger \& Rentschler, 1996; Strasburger, Rentschler, \& Harvey, 1994) and as a diagnostic tool (Beck, Diehl, Cleary, \& Group, 1993; Blommaert \& Timmers, 1987; Brown, Doran, \& Woodhouse, 1987; France \& France, 1988; Pelli, Robson, \& Wilkins, 1988; Rabin \& Wicks, 1996; Regan, 1988a, 1988b; Regan \& Neima, 1983a, 1983b). Low-contrast letter recognition has been studied in dyslexia, pediatric ophthalmology, amblyopia, glaucoma and ocular hypertension, diabetic and other retinopathies, cataracts, multiple sclerosis, Parkinson's disease (see Regan, 1988a, for a partial review and clinical relevance),

This study was supported by the Deutsche Forschungsgemeinschaft (DFG) Grants Str 354/2-1 to H.S. and Re 337/10-1 to Ingo Rentschler. Part of the study was conducted during a guest stay at the Psychology Department of the University of Colorado at Boulder. Particular thanks go to Lewis O. Harvey Jr. for posing the question under study and his continuous support in solving it, to Jon Roberts in the Boulder Psychology Department for his long-lasting help with Macintosh and DEC computer technology, to Bernhard Treutwein, who has been a knowledgeable discussion partner for all statistical and technical questions and a source for statistical literature, to Stan Klein for several rather thorough readings of the manuscript, and to Ingo Rentschler for his generous support. Correspondence concerning this article should be addressed to H. Strasburger, Generation Research Program (Bad Tölz), Human Studies Center, University of München, Goethestr. 31, D-80336 München, Germany (e-mail: hans.strasburger@1rz.uni-muenchen.de; http://www.grp.hwz. uni-muenchen.de). and also for exceptional vision (Rabin, 1995). It is a tool for studying lateral interaction or the crowding phenomenon (Strasburger et al., 1991). Few studies as yet have studied low-contrast letter recognition in peripheral vision (Herse \& Bedell, 1989; Higgins et al., 1996; Mackeben, 1999; Strasburger et al., 1991; Strasburger \& Rentschler, 1996; Strasburger et al., 1994). There is, thus, an accumulating body of data on the recognition of characters at low contrast in foveal and in disturbed vision, and it is time to explore the full visual field.

When we characterize pattern recognition across the visual field, we usually do so by a detailed description of foveal vision, together with a set of rules of how those foveal relationships generalize to peripheral viewing locations. As an example, the cortical magnification hypothesis predicts that performance varies across the visual field according to the neural projection scale in the retino-cortical pathway (Rovamo, Virsu, \& Näsänen, 1978; Virsu, Näsänen, \& Osmoviita, 1987). More generally, spatial-scaling approaches predict peripheral performance on the basis of neural densities (receptor, ganglion cell density, etc.) or psychophysical size-related measures like acuity, the minimal angle of resolution (Drasdo, 1991; Levi \& Klein, 1985; Weymouth, 1958). How to best describe foveal character recognition is itself an open question. Computational vision approaches argue that current channel models do not capture what is essential about the process of recognition, which would distinguish it from a (simpler) process of detecting image components (Barth, Zetzsche, \& Rentschler, 1998; Zetzsche \& Barth, 1990; see also Watanabe, 1985). Still, most psychophysical empirical results seem to conform well with a channel approach 
and provide a link between pattern detection and pattern recognition (Alexander, Xie, \& Derlacki, 1994; Harvey \& Gervais, 1978; Higgins et al., 1996; Solomon \& Pelli, 1994; Thomas, 1987).

By studying the contrast threshold for the recognition of characters, we have recently shown that character recognition varies across the visual field in a manner different from grating detection performance (Strasburger et al., 1991; Strasburger \& Rentschler, 1996; Strasburger et al., 1994) Related to that, spatial-scaling models, including the cortical magnification model, fail to account for these findings (Strasburger et al., 1994) and need to be replaced by a generalized model (Strasburger $\&$ Rentschler, 1996). The empirical link at the fovea between detection and recognition provided by the work of Harvey, Thomas, and others, cited above, might thus break down outside the fovea-that is, at eccentricities as low as, perhaps, $4^{\circ}$ of visual angle. The crucial roles seem to be played by pattern contrast and/or by the dimensionality of decision space, the noted discrepancies occurring with large characters of contrast less than unity (i.e., when patterns are not black and white) and when true recognition tasks rather than (few-alternative) discrimination tasks are considered (Strasburger \& Rentschler, 1996).

To more fully understand why pattern recognition contrast thresholds differ from those for detection tasks, we have studied the psychometric function that links performance to pattern contrast (Figure 1A). The threshold is just one point on that function, and a description of performance based solely on thresholds is incomplete without knowledge of the psychometric function's shape for the stimulus property under question. This becomes particularly apparent when thresholds are to be compared across different studies: The definition of threshold varies widely between studies, being set at the psychometric function's steepest point or at some criterion perfor-
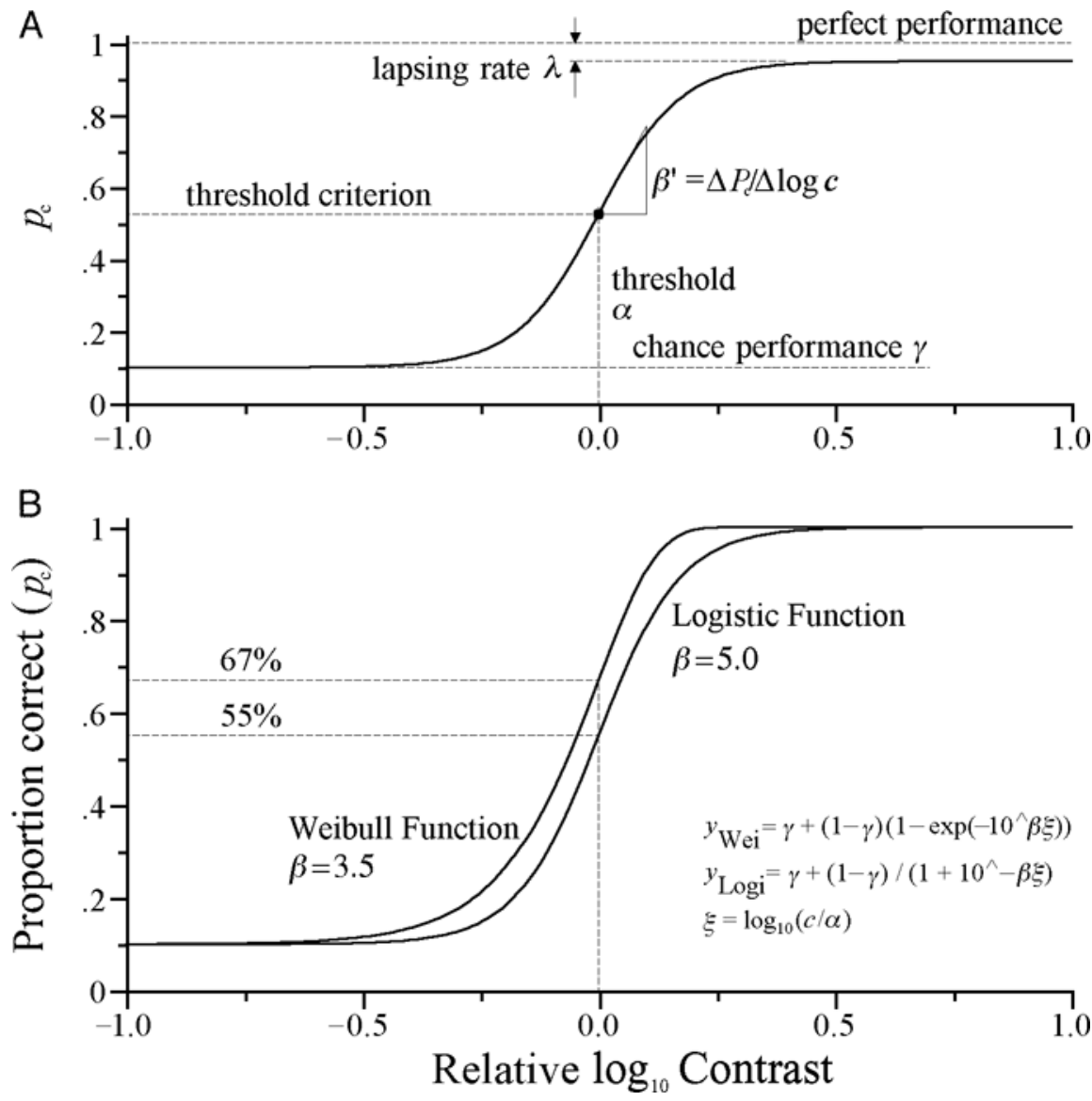

Figure 1. The psychometric function (in detection tasks sometimes also called frequency-ofseeing curve). (A) Definition of terms: abscissa, a measure of stimulus level [shown here is the logarithm of relative contrast, $\log (x / \alpha)$ ]; ordinate, subject performance as proportion $\boldsymbol{P}_{\mathrm{c}}$ of correct responses; $\gamma$, guessing rate; $\lambda$, lapsing rate; $\alpha$, the threshold, defined here as the point of inflection; $\Delta \boldsymbol{P}_{\mathrm{c}} / \Delta \log c$, slope on a $\log$ scale, its maximum denoted by $\beta^{\prime}$ here. (B) Analy tic forms of the two psychometric functions used in our fits, plotted here on a $\log _{10}$ scale. Slope is governed by parameter $\beta$; different values are needed between the functions for same slope (see the Method section). 
mance between $50 \%$ and $90 \%$, depending on the number of alternatives in the $n$-alternative forced-choice task ( $n$ AFC), the chosen psychometric function's analytic representation, convention, and taste. Thresholds thus mean different things in different studies, and for their quantitative comparison, the psychometric function and, particularly, its slope must generally be known.

Figure 1 shows a psychometric function in a forcedchoice task and explains the terms used here. The function plots subject performance as proportion $P_{\mathrm{c}}$ of correct responding as depending on stimulus level - that is, pattern contrast $c$. Guessing rate $\lambda$ corresponds to chance performance and depends on the number of choice alternatives; lapsing rate $\lambda$ (set to zero in the following for simplicity) quantifies response errors despite good visibility. Threshold $\alpha$ specifies the function's horizontal position and is defined here as the point of inflection - that is, the point of maximum slope or the point where the second derivative is zero. $\Delta P_{\mathrm{c}} / \Delta \log x$ is the slope on a log scale of stimulus level. Its maximum (which occurs at threshold) is denoted by $\beta^{\prime}$ here to show that it is related to the slope parameter (commonly denoted by $\beta$ ) in the psychometric function's analytic representation (see the Method Section and Strasburger, 2001a, for further details).

Of the four parameters that specify a psychometric function (Treutwein \& Strasburger, 1999), each plays a different role. Threshold is usually the most sensitive; it can be viewed as defining and bounding the system's operating range. The role of slope is usually seen as its being a measure of reliability, both of the measurement of threshold and of the system under investigation. A reduced slope might prove to serve as a diagnostic of a system malfunction (Lelkens \& Opponeer, 1983). Guessing and lapsing rate play a more technical role: The guessing rate is usually fully given by the task design and is, thus, in the experimenter's hand, and the influence of the lapsing rate is often negligible (except for 2AFC tasks; see Green, 1995). ${ }^{1}$

With regard to a theoretical understanding of the psychometric function's shape and how to interpret variations of its slope, there are few concepts. For the case of detection tasks, there has been extensive theoretical research on the psychometric function's meaning, in both the acoustic and the visual domains (e.g., Lelkens \& Opponeer, 1983; Nachmias, 1981). Individual sensory neurons show response functions (firing rate vs. stimulus intensity) that closely resemble a psychometric function, and one approach to interpreting psychophysical data is to model probability summation among sensory units (Graham \& Robson, 1987; Mayer \& Tyler, 1986; Pelli, 1985, 1987). For the case of pattern recognition (again, in both the acoustic and the visual domains), we are far from such psychometric function modeling. The reason is that it is as yet unclear in which way to model the combination of low-level sensory information in the pattern recognition process (Watanabe, 1985). A purely empirical, descriptive standpoint is therefore taken here, without attempting to give meaning to the psychometric function's shape.
The study of character recognition at low pattern contrast represents a fairly new area, and in order to have confidence in the slope estimates presented here, they were compared with those of other studies investigating the role of pattern contrast. Comparison of slope across studies, however, presents a particular problem: There is a flurry of slope measures that-although being mostly convertible among each other-make comparisons tedious and error prone, thus obscuring the problems of interest. Example measures in use are the slope or spread parameters in the chosen analytic representation (Weibull/Quick, logistic, Gaussian, hyperbolic tangent, each in log or linear scaling on the abscissa) or those that are data based, such as the 10\%-90\% range or the interquartile range. Adding to the confusion is the fact that there are three popularcontrast measures to choose from: Michelson contrast, the Weber fraction, or the ill-defined $\mathrm{dB}$ as used in perimetry (which goes "backwards" and has no meaningful anchor). To help in comparing slope results from different studies, relationships between a number of slope measures were derived (Strasburger, 2001), including the slope parameter in analytical representations such as the Weibull, logistic, cumulative normal, and other functions, the deci$\log$ unit, empirical measures such as the interquartile range, and slope in a $d^{\prime}$ representation. It is shown there that a particularly useful measure is the maximum slope (the slope at the point of inflection), for a number of reasons: The analytical functions in question are highly similar when equated for equal maximum slope. Maximum slope has a simple physical interpretation, being the highest increment of the proportion of correct responses per log-unit increase on the stimulus scale. Its value, specified together with physical units, can thus be compared universally, including tasks from different sensory modalities. And last, the value can be easily derivedonce the function is fitted - by the formulas given in Strasburger (2001).

The goal of this report is to study the variation of the slope of the psychometric function for character recognition versus pattern contrast, across the horizontal meridian, for binocular and monocular vision. A fine, $2^{\circ}$ raster of horizontal eccentricities is used across the entire range to obtain a detailed description of any variation. The slope data at finely spaced retinal positions presented here should provide a firm basis on which to build future studies.

\section{METHOD}

\section{Stimuli}

Single-digit stimuli, in a plain font without serifs (Figure 2), were presented on a video processing system using a $512 \times 512 \times 8$ image and a high-precision video attenuator that achieved approximately 15 bits of gray-scale resolution (see Bach, Meigen, \& Strasburger, 1997, for a short overview of high-contrast resolution techniques). This equipment was the same as that used previously and is more fully described elsewhere (Strasburger, 1996, 2002; Strasburger et al., 1991). The current software version, named R_Contrast, runs on standard equipment and is described in Strasburger (1997). Numeric characters were presented singly, for $100 \mathrm{msec}$, as white patterns against a $62 \mathrm{~cd} / \mathrm{m}^{2}$ background on a high-precision 13-in. 
black-and-white raster CRT (Barco TVM 3/37). Stimuli were composed of at least $25 \times 35$ pixels and had a physical size greater than $11 \mathrm{~mm}$ in height in order to eliminate image blur caused by the bandwidth limitations of the video signal attenuator. We had found that visual performance suffered with that setup when the physical size of the stimuli was less than these values (Strasburger et al., 1991).

For stimulus sizes below $1^{\circ}$, the retinal size was manipulated by varying viewing distance from $64 \mathrm{~cm}$ to $10 \mathrm{~m}$. Pilot testing showed that, in this range, viewing distance in and of itself has no effect on recognition thresholds. Changing viewing distance is thus a simple and effective way of changing retinal image size. At viewing distances closer than $64 \mathrm{~cm}$, however, accomodative error and the visibility of the raster lines begin to effect the contrast threshold (Strasburger, 1996, 2002). So, for retinal image sizes larger than $1^{\circ}$, viewing distance was kept constant at $64 \mathrm{~cm}$, and the physical size of the stimulus on the screen was varied.

Stimulus contrast was measured in $\log _{10}$ Michelson units- that is,

$$
\log _{10} c=\log _{10}\left(l_{t}-l_{b}\right) /\left(l_{t}+l_{b}\right),
$$

with $l_{t}, l_{b}$ being the luminances of target and background in $\mathrm{cd} / \mathrm{m}^{2}$, respectively. To allow for pooling data across conditions of different threshold, contrast was specified relative to the threshold $\alpha$ at the given condition:

$$
\xi=\log _{10} c_{\text {rel }}=\log _{10}(c / \alpha)=\log _{10} c-\log _{10} \alpha .
$$

Absolute contrast $c$ can be obtained by inverting Equation 2,

$$
\log _{10} c=\xi+\log _{10} \alpha .
$$

and inserting the appropriate threshold value $\alpha$ for a specific condition.

In comparing contrast results between different studies, note that an alternative contrast measure-the Weber fraction-is often used:

$$
c_{W}=\left(l_{t}-l_{b}\right) / l_{b} .
$$

The latter is nonlinearly related to the Michelson measure, the relation given by

$$
c_{M}=c_{W} /\left(2+c_{W}\right) .
$$

In converting to Weber units, the abscissas in the following graphs would therefore undergo a nonlinear transformation. For low threshold contrast, the nonlinearity is not pronounced, however, and the relative measures are about equal:

$$
\begin{gathered}
c_{\text {rel } M} \approx c_{\text {rel } W} \quad\left|c_{M}\right| \leq 0.1 . \\
c_{M} \approx c_{W} / 2
\end{gathered}
$$

\section{Subjects}

Two observers ( 1 male and 1 female, both 25 years of age) served as subjects in this experiment. Both had normal vision and wore no corrective lenses during the experiments. They were naive as to the purpose of the experiment. Each subject made approximately 30,000 judgments over a period of 1 year.

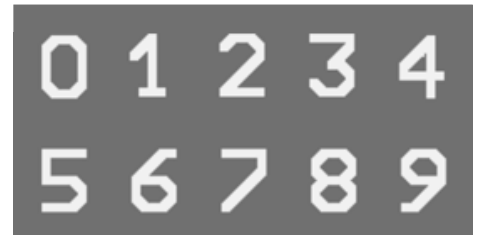

Figure 2. The stimulus set, shown at some intermediate contrast.

\section{Procedure}

Response data were acquired in an adaptive, maximum-likelihood staircase procedure, ML-PEST (Harvey, 1986, 1997; see Treutwein, 1995, for a review), with stimulus contrast as the adaptive variable. In effect, the adaptive algorithms quickly converge to the threshold, thus concentrating response data around the latter; Figure 3A shows a sample run of 30 trials. Adaptive procedures, owing to that property, are ideal for threshold estimation, and even though a somewhat higher spread of data will be the most efficient for slope estimation, data from the adaptive procedures are still well suited for reliable slope estimation and are reported to be superior to the method of constant stimuli (Leek, Hanna, \& Marshall, 1992). Recently, adaptive principles have been extended so as to be optimized for slope estimation (King-Smith \& Rose, 1997; Kontsevich \& Tyler, 1999).

Measurements were organized into blocks of trials having fixed size and retinal locus, with stimulus contrast selected anew after each trial on the basis of the maximum-likelihood estimate of the contrast threshold (physical contrast resolution, 1/80 log unit). The likelihood is obtained from a fit of a Weibull function of fixed shape $(\beta=3.5$, $\lambda=.10, \lambda=0)$. Since the function only influences stimulus presentation strategy, it does not predetermine which psychometric function will later fit the data best. A block was ended when the $95 \%$ confidence interval for the estimated threshold was $\leq 0.2 \log$ units, which required approximately 30 trials. The threshold was obtained from the final fit in a block. Threshold for each condition was measured twice in a counterbalanced order. The $95 \%$ conf idence interval of the mean of the two measurements is approximately $0.2 / \sqrt{2}=0.15$ $\log$ units (since the log-likelihood function is asymptotically normal around its maximum [Morgan, 1992] and confidence intervals thus vary with the square root of the number of trials). The results of each trial-that is, stimulus contrast, digit presented, and observer response-were saved for detailed analysis.

Twenty-one retinal loci on the horizontal meridian, ranging from $0^{\circ}$ to $38^{\circ}$ eccentricity in the left and right visual field, were tested with up to 22 stimulus sizes ranging from $0.07^{\circ}$ to $4.8^{\circ}$. Thresholds were obtained separately for each condition, and the ratio of contrast to contrast threshold calculated to obtain relative contrast according to Equation 2. Each value $\xi$ in the following thus represents the $\log$ contrast increase over the log threshold in that specif ic run of $30+$ trials at a specific size-eccentricity condition. Two viewing conditions were used: binocular and monocular. With monocular viewing, the stimuli were presented in the temporal visual field.

\section{Data Pooling}

At each retinal location, the (binary) response data for relative contrast were pooled across stimulus size in order to increase the reliability of slope estimation. Each slope estimate thus refers to the set of visible stimulus sizes at a given retinal eccentricity, not to individual sizes. Note that pooling of raw binary data (unlike pooling of probability data) has a straightforward mathematical basis and, indeed, underlies all psychometric testing. Some kind of raw data pooling - across runs, subjects, or some other variable — is required for a reliable estimate. Pooling across sizes was chosen for two reasons: First, I was interested in intraindividual effects, and repeated runs at fixed size-eccentricity conditions intolerably increase the observer time (for the present conditions, the subjects spent 1 man-year of paid observing time). Second, since the $M$-scaling principle is not valid for low-contrast character recognition (Strasburger et al., 1994), there is no natural way of choosing a single fixed size at a given eccentricity, so that it would be directly comparable with that at another eccentricity. Thus, the study of how slope depends on stimulus size in the visual field is left for further research. As an aside, many more data-about tenfold — are needed for reliable slope estimation than for threshold estimation; intuitively, this can be understood by noting that the slope corresponds to the first derivative 


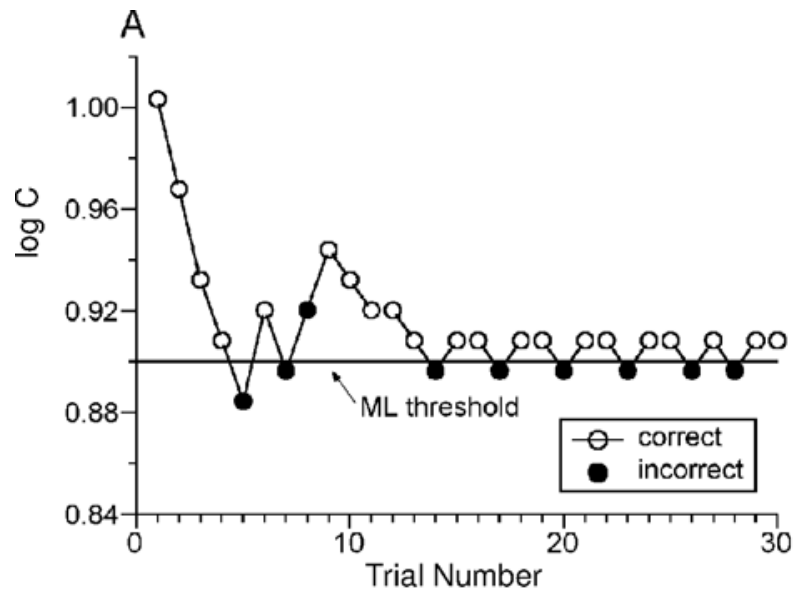

B

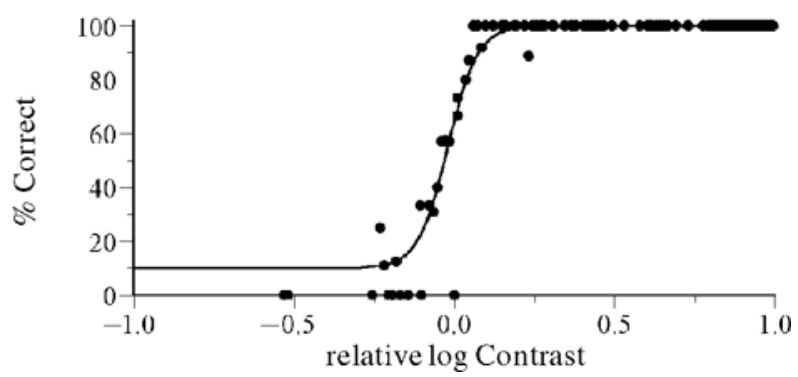

Figure 3. (A) A sample run of 30 trials with a threshold estimate of 0.9 log Michelson contrast. (B) A sample fit to 590 responses.

of a function (and threshold to a point on the function itself) and that estimates of derivatives are inherently more noisy.

\section{Curve Fitting}

Two sigmoid functions of slightly different shape were used to fit the data, to test which function would fit better. The Weibull function was chosen for its popularity in contrast detection experiments, so that the results shown here could be compared with those; the logistic function was included because of its rotation symmetric shape. On linear coordinates, the functions are given by

$$
P_{\mathrm{c}}(c)=\gamma+(1-\gamma)\left(1-\frac{1}{e^{(c / \alpha)^{\beta}}}\right) \quad \text { (Weibull function) }
$$

and

$$
P_{\mathrm{c}}(c)=\gamma+(1-\gamma)\left(\frac{1}{1+\frac{1}{(c / \alpha)^{\beta}}}\right), \quad \text { (logistic function) }
$$

with parameter definitions as in Figure 1A. Equations 7 and 8 can be rewritten as

$$
\begin{aligned}
& P(c)=\gamma+(1-\gamma)\left(1-e^{-s}\right) \quad \text { with } \quad s=e^{\beta \xi}, \\
& \text { (Weibull function) } \\
& P(c)=\gamma+(1-\gamma)\left(1+e^{-s}\right)^{-1} \quad \text { with } \quad s=\beta \xi,
\end{aligned}
$$

(logistic function) and

$$
\xi_{e}=\log _{e}(c / \alpha)=\log _{e}(c)-\log _{e}(\alpha),
$$

in the way they were used in the fits and so that the natural logarithm $\log _{e}(c)$ of the physical variable appears as term. Figure $1 \mathrm{~B}$ shows the two functions plotted on a $\log _{10}$-contrast abscissa. The point of inflection in both cases is at $x=\alpha$. The logistic function is rotation symmetric about that point; it is thus halfway up (at 0.55 when $\lambda=.1$ ). For a Weibull function with $\lambda=.1$, the point of inflection is at 0.67 . When the functions are plotted relative to the threshold, they therefore appear shifted against each other. Note that $\alpha$ and $\beta$ will have different numerical values for functions of similar shape, and caution must be exercised in comparing logistic parameters with Weibull parameters. The steepness of the two functions may be meaningfully compared in terms of the maximum slope, $\max \left(\Delta P_{\mathrm{c}} / \Delta \log c\right)$, which for the two functions occurs at the threshold $\alpha$ and is denoted by $\beta$ here. This value is easily computed from $\beta$ (see Strasburger, 2001a, for conversion formulas, and Klein, 2001, for the relationship to linear abscissa).

The maximum-likelihood estimates of both $\alpha$ and $\beta$, for both logistic and Weibull functions, were computed from the data, holding $\lambda$ constant at 0.1 (and the lapsing rate $\lambda$ at a small value, $0.01 \%$; see note 1), using a maximum-likelihoo d curve-fitting program, MLPFIT, written by L. O. Harvey $(1986,1997){ }^{2}$ The maximum-likelihood fitting procedure has a well-developed statistical basis (Collett, 1991; Morgan, 1992; Collett gives an exceptionally clear presentation of binary-data analysis) and is more useful in this context than the oftenused chi-square minimization or linear regression of probit values, as has already been discussed by Finney (1971).

Maximum-likelihood fitting (like chi-square minimization) operates on the raw binary data, not on percentage-correct data. One advantage of this is that marginal values (0\% and $100 \%)$ are handled naturally. An advantage over chi-square is that equally and nonequally spaced stimulus levels (contrasts), as well as equal and unequal numbers of stimuli at any given level and in particular large numbers of single responses (at a given level), are handled equally well. For the purpose of estimating response probabilities at each stimulus level (but not for fitting), responses were also combined into bins of relative contrast and are shown as such in the graphs.

Figure 3B shows a sample fit. Twenty runs of, at the mean, 29.5 trials each (i.e., a total of 590 responses), with identical stimulus conditions (foveal presentation, stimulus size $1^{\circ}$, young emmetropic subject) were pooled as described, and a logistic function was fit to the response data. Thresholds varied between -2.05 and $-1.90 \mathrm{log}$ Michelson contrast, and the fit was to relative-to-threshold contrast values. The resulting slope parameter beta was 9.42 , with the $95 \%$ confidence interval ranging from 6.68 to 12.0. Threshold estimate alpha (not surprisingly) was close to zero $(-0.03)$. Single responses are seen as points at the marginal percentages, $100 \%$ (correct) or $0 \%$ (incorrect); levels at which several responses are obtained give intermediate percentage correct values. Note that every sequence was started at the same, above-threshold contrast of 0.1 , so there are many responses on the top right of the graph.

\section{RESULTS}

The effects of stimulus size, retinal eccentricity, and viewing condition on contrast threshold in the present experiments were virtually identical to the effects previously reported (Strasburger et al., 1991; Strasburger et al., 1994). The focus of our analysis will therefore be on the shape of the psychometric functions under the different experimental conditions.

\section{Binocular Viewing, Summary Fit}

The probability of a correct response as a function of $\log$ relative contrast is plotted in Figure 4 for Subject W.B. 
under binocular viewing conditions. For an overall fit, the data for all sizes and eccentricities were pooled. The data have been obtained under a wide range of stimulus sizes and visual eccentricities. Sizes were those visible from a set of 19 , ranging from $0.1^{\circ}$ to $4.8^{\circ}$ (from $0.1^{\circ}$ to $1.2^{\circ}$ in steps of $0.1^{\circ}$ plus $\left.1.6^{\circ}, 2^{\circ}, 2.4^{\circ}, 2.8^{\circ}, 3.2^{\circ}, 4^{\circ}, 4.8^{\circ}\right)$; eccentricities were in steps of $2^{\circ}$ in the full range of recognition, from $0^{\circ}$ to $38^{\circ}$ eccentricity, on both the left and the right horizontal meridian. Fine spacing in retinal eccentricity was aimed at to provide confidence that local variations would not go unnoticed by course sampling. The blind spot was included in the analysis of the effect of eccentricity (below) as a counterexample to homogeneous processing but was excluded from the overall fit. In total, there were 530 different size/field-position combinations. The relative contrast was computed with Equation 2, using the threshold measured under the appropriate size and visual field position.

Figures $4 \mathrm{~A}$ and $4 \mathrm{C}$ show how the adaptive algorithm has allocated the testing contrast relative to the threshold. The datapoints show response data grouped into small bins of constant $0.01-\log$-units width and are located at the mean of that bin. In Figure 4A, the number of responses is coded as both needle length and dot size, with large dots representing more than 50 and up to several thousand responses per point. The majority of stimulus values is clustered where the psychometric function departs from its asymptotes, with peak density occurring at the threshold. As a function of contrast (Figure 4C), response density has a sharp peak: The maximum is 3,442 responses per 0.01 $\log$-unit bin, the number of stimuli dropping below $5 \%$ at $0.1 \mathrm{log}$ units (ratio, 1.25) away from threshold and below $0.1 \%$ at $0.25 \log$ units below or $0.18 \log$ units above threshold (contrast ratios, 1.5 and 1.8, respectively). Figure $4 \mathrm{~A}$ shows that stimulus values are distributed much less narrowly along the psychometric function trajectory, showing an approximately Gaussian distribution there. The distribution seems thus well suited to estimate both thresholds and psychometric function slopes. In Figure 4B, responses were grouped differently, into bins of 50 responses each, in an attempt to visualize the number of responses as number of datapoints, but many datapoints are not visible, since they coincide fully, especially for contrasts near the measured threshold.

Figures $4 \mathrm{~A}$ and $4 \mathrm{~B}$ show the overall best-fitting logistic function, its parameters computed by maximum-likelihood fitting to the binary data, using MLPFIT. The maximumlikelihood values of $\alpha$ and $\beta$ for the logistic and the Weibull functions are shown in Table 1. Parameter $\alpha$ comes out close to zero, as was expected; the maximum slope $\beta^{\prime}$ for the two functions is 4.84 and $4.14 p_{c} / \log _{10}$, respectively, and the corresponding steepness parameters are $\beta=9.35$ for the logistic and $\beta=5.43$ for the Weibull functions. The goodness-of-fit measure $\chi^{2}$ (data binned by unique stimulus values) and the degrees of freedom (number of unique stimulus values minus one) are also given in Table 1.

The summary fits for the 2nd subject (C.H.) give very similar results. Viewing was again binocular on the left and right horizontal meridian, with sizes, with a few exceptions, being the same as those for Subject W.B.; eccentricities were from $0^{\circ}$ to $18^{\circ}$ in steps of $2^{\circ}$ (the binary response data above $18^{\circ}$ were lost owing to equipment failure). The results are shown in Table 1 and Figure 5. The overall slopes (e.g., $\beta^{\prime}=4.99 p_{c} / \log _{10} c$ for the logistic function) are almost identical to those for the 1 st subject.

\section{Effect of Retinal Eccentricity}

We now ask whether the psychometric function varies across eccentricity. From Figure 4, it is evident that performance, in almost all cases, reaches $100 \%$, and chance correct probability is .1 by design. So, the question reduces to the one of whether slope varies with eccentricity. The analysis described in the preceding section was repeated for each eccentricity separately, with data collapsed between left and right visual fields and between different stimulus sizes. The independent variable is again normalized contrast.

These contrast thresholds for normalization are summarily shown in Figure 6 (see Strasburger et al., 1994, for details). (Data in the blind spot are replaced by interpolated data in the figure in order not to divert from the overall characteristics, but the actual data are used for the normalization.) Interestingly, foveal thresholds increase slightly toward larger characters above an optimum size, similar to the slight low-frequency drop-off of a contrast sensitivity function, so that, for this subject, recognition performance for large letters is optimal at slightly eccentric positions $\left(5^{\circ}\right)$. Most important, however, threshold for large character sizes increases with an eccentricity above $5^{\circ}$, and the increase is not compensated by any size scaling that one might apply, in violation of the cortical magnification concept, as we have shown previously (Strasburger \& Rentschler, 1996; Strasburger et al., 1994; possible reasons are discussed in Strasburger, 2002).

Table 2 shows the results of that slope analysis-slopes and slope confidence intervals for fits of the logistic and the Weibull functions separately for each eccentricity-and Figure 7A plots maximum slope $\beta^{\prime}$ as a function of eccentricity. The table's first row repeats the summary data from Table 1. Slopes in the table are given both as $\beta$ values and as maximum (i.e., point of inflection) slopes, to ease comparison with other studies. For comparing the logistic with the Weibull function, only the maximum slopes are meaningful (column 5 and 6; see Strasburger, 2001, for the conversions). Since confidence intervals were repeatedly determined, Bonferroni-corrected intervals are given in column 8 (see below). The significance markings (column 9) refer to the latter.

Of the 17 eccentricities up to $32^{\circ}$, only three differ significantly from the overall value in row 1 : those at $12^{\circ}, 20^{\circ}$, and $32^{\circ}$. The blind spot for this subject is at $14^{\circ}-18^{\circ}$, as determined by static perimetry (Strasburger \& Rentschler, 1996 , Figure 3 ), and the locations at $12^{\circ}$ and $20^{\circ}$ are thus adjacent to it. Viewing in the blind spot is monocular, and contrast thresholds are increased by about a factor of two. Thus, at the blind spot's border, two different visual pro- 


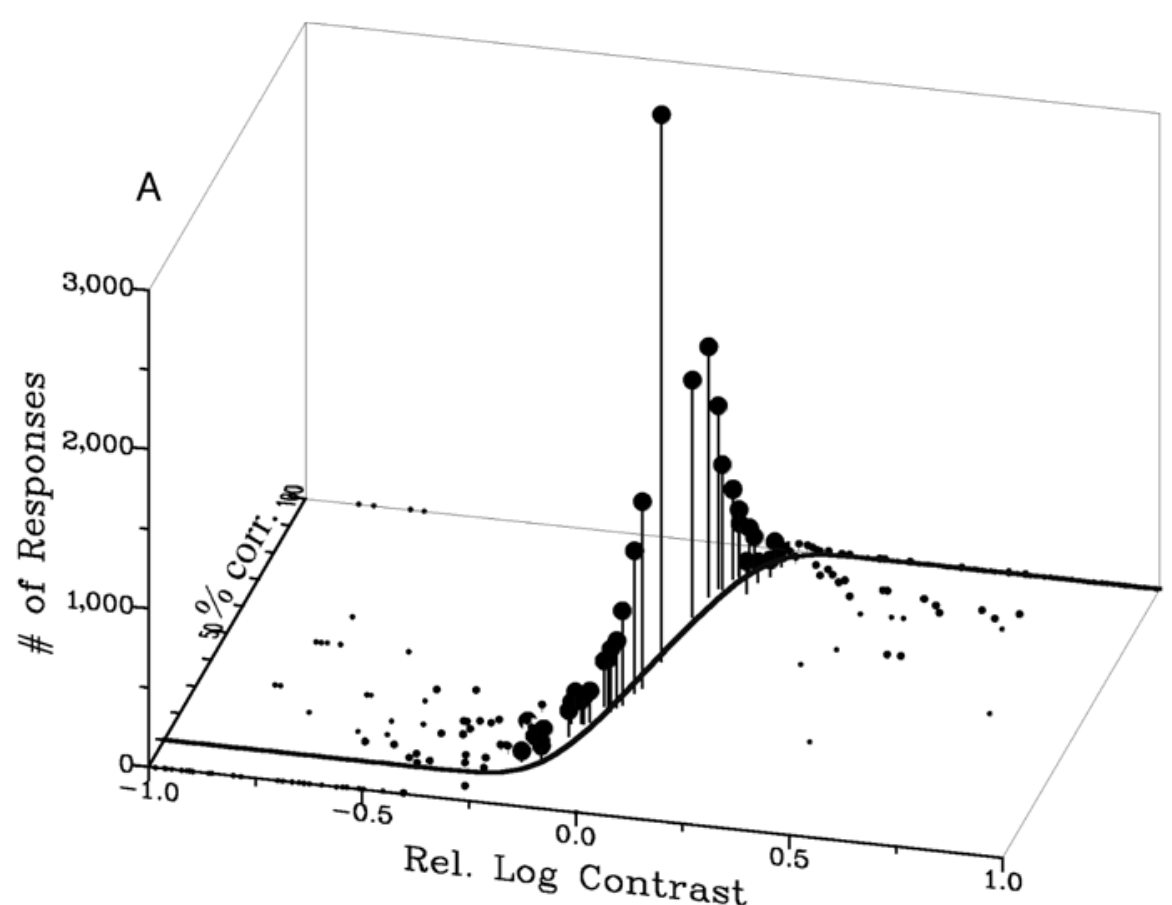

B
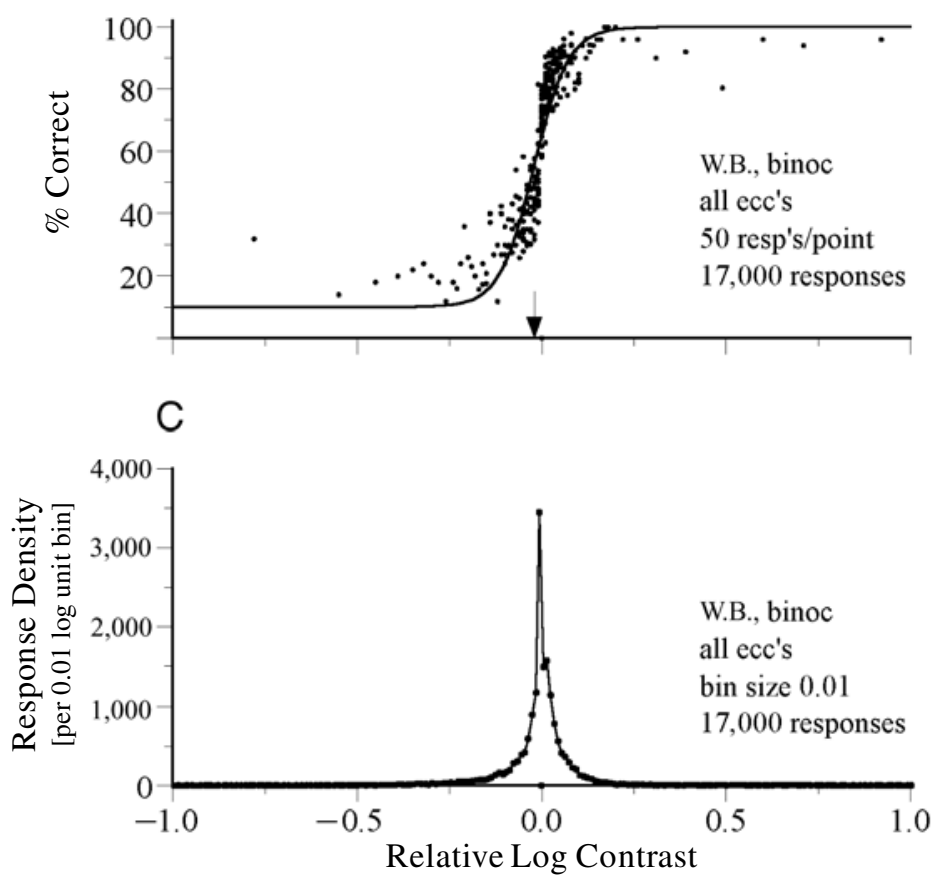

Figure 4. Summary psychometric function and subject responses for binocular viewing of digits on the horizontal meridian, with response data pooled over all stimulus sizes and eccentricities. Around 17,000 responses are included in the graph. (A) Three-dimensional representation to show the overall distribution of responses. Each datapoint represents a contrast bin of $0.01 \mathrm{log}$ units width and is located at the mean of that bin. The number of responses is coded as both needle length and dot size. Large dots, $\geq 50$ responses per point; medium size dots, $6-50$ responses/point; small dots, 1-4 responses/point. (B) The maximum likelihood-fitted logistic function has a maximum slope of $\beta^{\prime}=4.84 p_{c} / \log _{10}$ unit. Responses are regrouped so that each datapoint represents a fixed number of 50 responses. Note that the curve fitting operates on the binary, not the grouped, data. (C) Number of responses versus normalized stimulus contrast. 
Table 1

Parameters of Psychometric Functions for Binocular Recognition of Singly Presented Characters on the Horizontal Meridian.

\begin{tabular}{|c|c|c|c|c|c|c|c|c|}
\hline Subject & Function & $\alpha$ & $\begin{array}{c}\beta \\
(\text { base } e)\end{array}$ & $\begin{array}{c}\gamma \\
\text { (fixed) }\end{array}$ & $\chi^{2}$ & $d f$ & $\begin{array}{l}95 \% \text { Confidence } \\
\text { Interval of } \beta\end{array}$ & $\begin{array}{c}\text { Maximum Slope } \\
\left(p_{\mathrm{c}} / \log _{10}\right) \\
\end{array}$ \\
\hline W.B. & logistic & -0.02 & 9.35 & .1 & 4,399 & 3,702 & $8.83 / 9.90$ & 4.84 \\
\hline $\begin{array}{l}(17,698 \text { responses, } \\
62.1 \% \text { correct })\end{array}$ & Weibull & 0.01 & 5.43 & .1 & 4,539 & 3,702 & $5.15 / 5.70$ & 4.14 \\
\hline C.H. & logistic & -0.018 & 9.64 & .1 & 2,803 & 2,173 & $9.04 / 10.25$ & 4.99 \\
\hline $\begin{array}{l}(6,231 \text { responses, } \\
65.2 \% \text { correct })\end{array}$ & Weibull & 0.01 & 5.53 & .1 & 2,990 & 2,173 & $5.18 / 5.88$ & 4.22 \\
\hline
\end{tabular}

Note-Maximum slope $\beta^{\prime}$ is derived from $\beta$ by $\beta^{\prime}=0.762, \beta_{\text {Wei }}=0.518 \beta_{\text {Logi }}\left(p_{\mathrm{c}} / 10_{\mathrm{c}}\right)$ for the $10 \mathrm{AFC}$.

cesses are acting, depending on the precise locus of fixation. This fact is the likely cause for the flattened psychometric function. We have no explanation for the slightly increased slope at $32^{\circ}$, except that we have fewer data at the more extreme eccentricities (form recognition is confined to the central $\pm 40^{\circ}$ ), and occasional misestimations toward higher slope then seem to occur more easily (Leek et al., 1992) and might not carry much meaning. Further out, at the far eccentric positions of $34^{\circ}$ and beyond, slope seems to be lower if we disregard the value at $40^{\circ}$ eccentricity, for which there are few data. A possible reason might be the high attentional strain required to make judgments under these conditions; only the largest targets were recognizable. Trials with strong and with weak attention might be mixed, giving rise to a flatter psychometric function. The open symbols in Figure 7A show the slopes obtained from fits of the Weibull function. Both functions give similar results, with no systematic difference visible.

The table also contains reliability estimates for the slopes. Based on an approximation $\chi^{2}=-2 \log L$ for the likelihood ratio $L$ (Hays, 1981), Harvey's MLPFIT determines a $95 \%$ confidence interval for $\beta$ by finding that point on the log-likelihoodfunction - as a function of $\alpha$ and $\beta$ where it drops to $\chi^{2} / 2$ below the maximum. Here, $\chi^{2}$ is set to 5.02 , the value that gives a $95 \%$ confidence interval with $1^{\circ}$ of freedom (refer to Harvey, 1986, 1997, for an excellent exposition; 5.02 is the one-tailed value corresponding to a probability of .025; since the confidence interval is twotailed, this value corresponds to a probability of .05). Since these confidence intervals are appropriate for obtaining a single estimate - that is, at one eccentricity - they need to
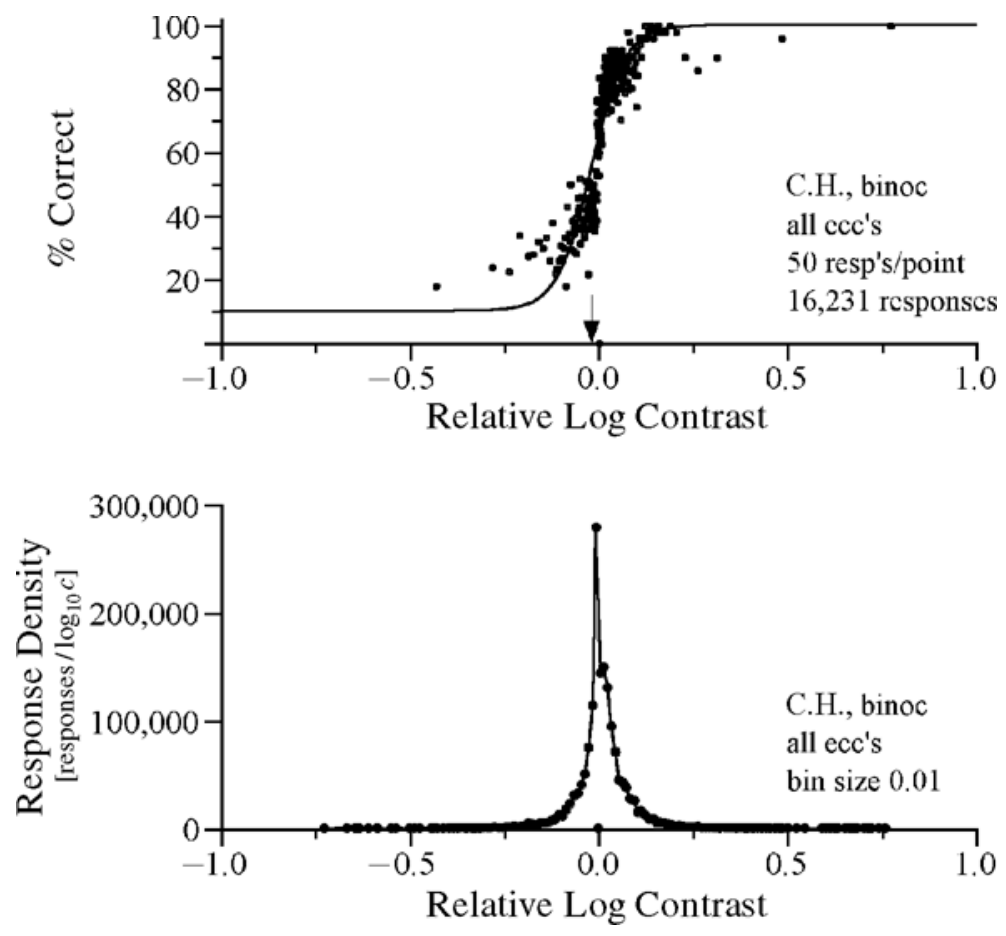

Figure 5. Summary psychometric function and subject responses for binocular viewing on the horizontal meridian (like Figure 4) for the 2nd subject. 


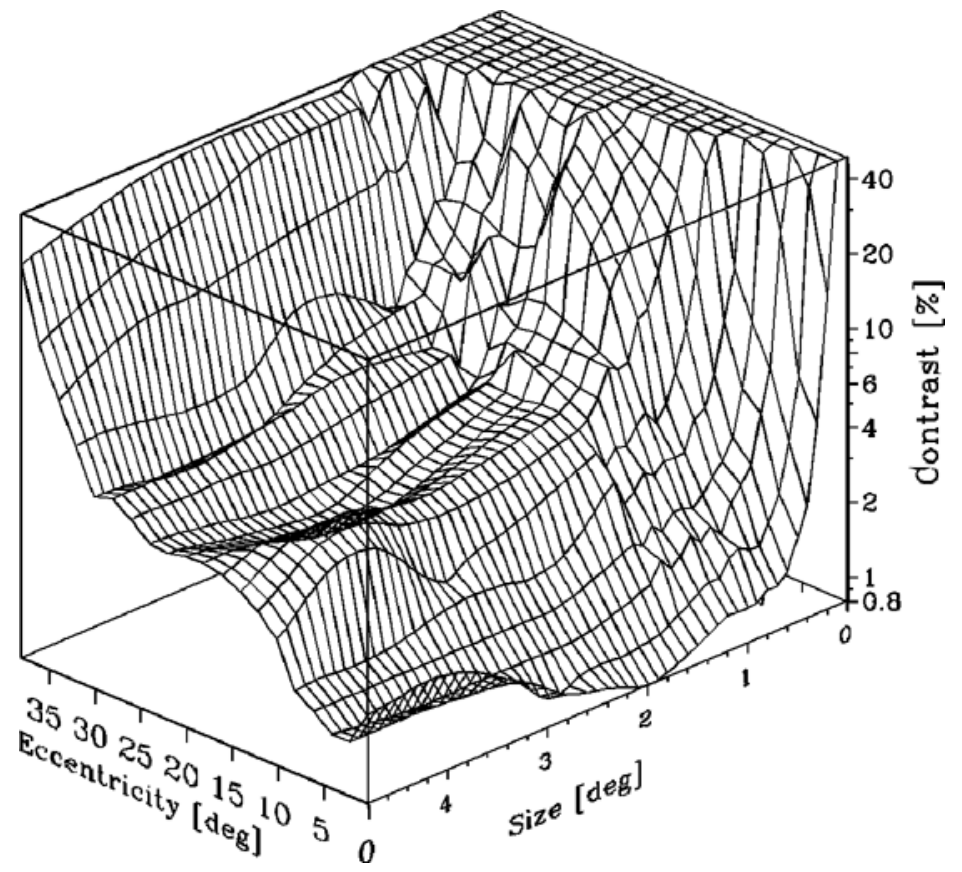

Figure 6. Recognition contrast thresholds for binocular viewing as a function of target size and eccentricity on the horizontal meridian. Mean of the left and right visual fields. Data in the blind spot are interpolated from binocularly visible neighboring positions. (From "Cortical Magnification Theory Fails to Predict Visual Recognition,” by H. Strasburger, I. Rentschler, and L. O. Harvey Jr., 1994, European Journal of Neuroscience, 6, p. 1584. Copyright 1994 by Blackwell Science. Adapted with permission.)

be Bonferroni corrected for repeated estimation, $n=21$ times in the table. We have calculated the $95 \%$ Bonferronicorrected intervals by setting the error rate to $5 \% / n$ to obtain a nominal overall error rate of $5 \%$ (see standard statistics book; e.g., Hays, 1981). The significance markings are based on these latter intervals.

Table 3 shows the results of the analysis across eccentricity for the 2 nd subject (C.H.), containing the $\beta$ values, the slopes at the point of inflection, and $\beta$ confidence intervals (for the conversions, see Strasburger, 2001a). The Bonferroni-corrected confidence intervals are based on a $5 \% / 10$ error rate (confidence interval widening by a factor of 1.425). The slopes and their confidence intervals are plotted in Figure 7B. There is little variation in the slopes of the logistic, with only the foveal function being slightly shallower. In the Weibull fits, there is a shallower slope for the two centralmost functions. Inspection of the response data and function shape showed, however, that these two fits were not good and seemed overly influenced by an increased lapsing rate that happened to occur in these two conditions (the lapsing rate was forced to $0.01 \%$ in the fits; see note 1). Therefore, the overall $\beta^{\prime}=5.0 p_{\mathrm{c}} / \log _{10}$ can be considered as representative for all eccentricities.

The field of recognition extends to about $40^{\circ}$ eccentricity on the horizontal meridian (Strasburger \& Rentschler,
1996). Summarizing, a slope of $\beta^{\prime}=4.8 p_{\mathrm{c}} / \log _{10}$ is characteristic for binocular viewing over a wide range of eccentricities on the horizontal meridian, extending to at least $32^{\circ}$, and there seems a slight decrease of slope near the border of the field of recognition.

\section{Monocular Viewing}

We finally ask whether the psychometric function is different for monocular viewing. The subject (W.B.) has repeated the same measurements as before, with both eyes separately, and on both the left and the right horizontal meridian at 12 different eccentricities between $0^{\circ}$ (foveal) and $36^{\circ}\left(0^{\circ}, 2^{\circ}, 4^{\circ}, 10^{\circ}, 12^{\circ}, 14^{\circ}, 16^{\circ}, 18^{\circ}, 20^{\circ}, 28^{\circ}, 32^{\circ}\right.$, $36^{\circ}$ ). There were thus four eye/field combinations (left eye/ left field, right eye/left field, etc.). Stimulus sizes were similar as in the binocular case $\left(0.2^{\circ}\right.$ or minimum visible to $1.6^{\circ}$ in steps of $0.1^{\circ}$ plus $\left.1.6^{\circ}, 2.4^{\circ}, 3.2^{\circ}, 4.0^{\circ}, 4.8^{\circ}\right)$. Nineteen thousand responses were collected overall.

The results on threshold (Strasburger, 1996, 2002) are only summarized here. The general finding was that monocular thresholds were higher than their binocular counterparts by about a factor of two. This can be characterized as full binocular summation; it is unlike the findings for grating detection, where summation is generally around $\sqrt{2}$ (Campbell \& Green, 1965), and can be de- 

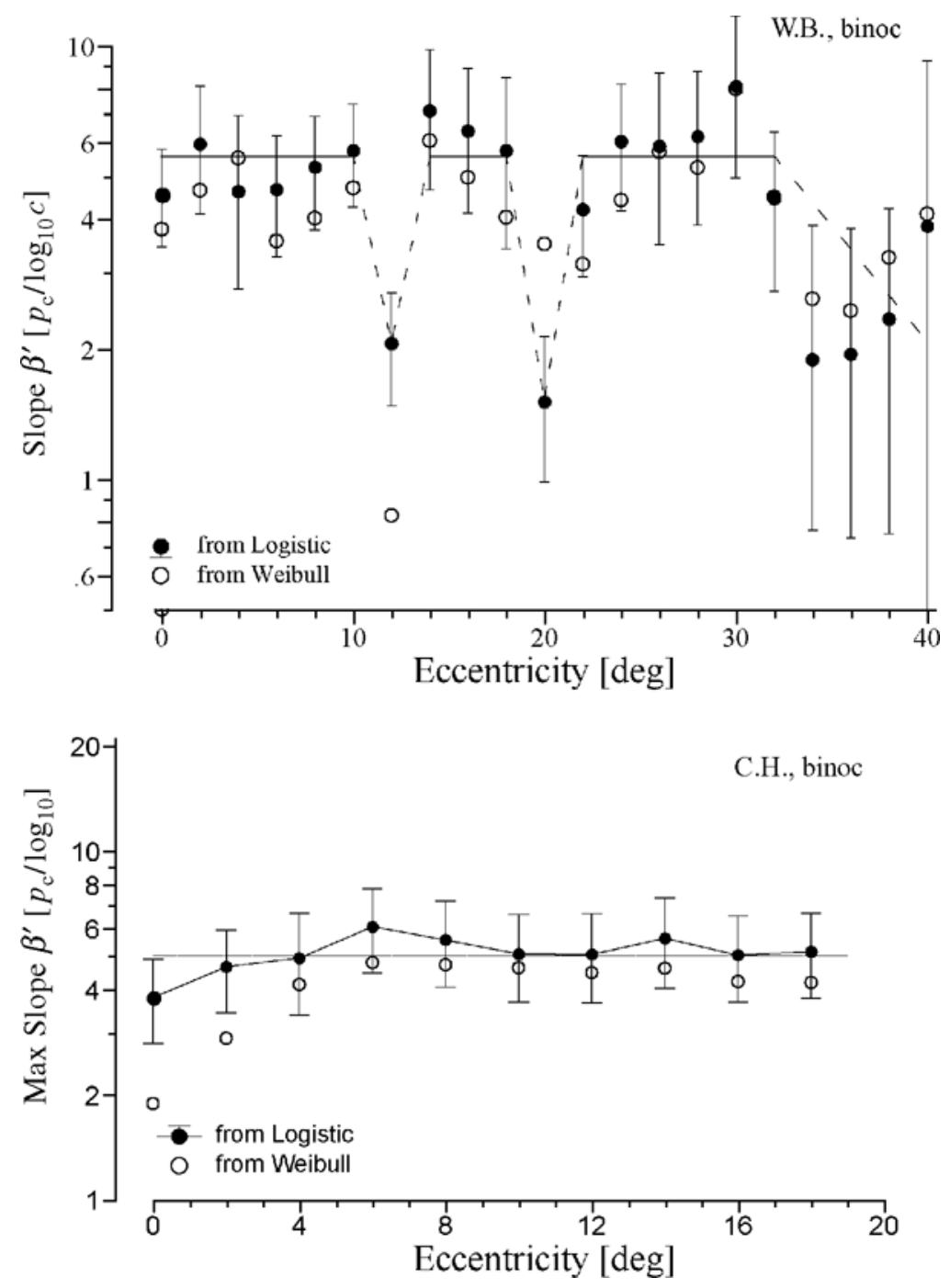

Figure 7. Slope $\beta^{\prime}$ at the point of inflection, derived from logistic function fits (filled symbols) and Weibull function fits (open symbols) at points of fixed retinal eccentricity on the horizontal meridian, for binocular viewing. (A) Results for Subject W.B. The blind spot is at $14^{\circ}-18^{\circ}$ eccentricity and the two points at $12^{\circ}$ and $20^{\circ}$ are at its borders. Slope is in units of proportion correct per $\log _{10}$ unit and is obtained by $\beta^{\prime}=(1 / 4)(1-\gamma)\left(\log _{\mathrm{e}} 10\right) \beta$ (see Strasburger, 2001). The error bars show Bonferroni-corrected confidence intervals for the logistic slopes. The horizontal line is drawn at $\beta^{\prime}=5.54 p_{\mathrm{c}} / \log _{10} c$, which is the geometric mean of the values for the logistic up to $32^{\circ}$, excluding the blind spot. (B) Results for Subject C.H.

scribed by probability summation. An interesting departure from the general factor-of-two summation occurs for nasal, but not temporal, vision at eccentricities between around $20^{\circ}$ and $30^{\circ}$. The monocular thresholds there increase up to the six-fold of the binocularvalue: Low-contrast recognition thus seems predominantly mediated by temporal vision in that region of the visual field.

Table 4 summarizes the results obtained from MLPFIT. The first line gives an overall fit; the logistic function's $\beta^{\prime}=4.43 / \log _{10}(\beta=8.56)$. Thus, there is no difference to the binocular case. Across eccentricity, slopes $\beta^{\prime}$ for the logistic function are, with one exception (at $28^{\circ}$ ), above
1.3 per $\log$ unit (i.e., $\beta \geq 6$ ), so the function is everywhere quite steep. There is some numerical variation in the slope parameter, but no clear trend. Figure 8 plots the slopes for the logistic and Weibull functions (leaving aside the point at $36^{\circ}$, for which there are few data). The horizontal line shows the overall slope of the logistic.

As in the binocularcase, the table lists the Bonferronicorrected $95 \%$ confidence intervals. On this level, four deviations are significant, at $2^{\circ}, 4^{\circ}, 28^{\circ}$, and $36^{\circ}$. Except for $36^{\circ}$ eccentricity, the estimates are obtained for a comparable amount of trials. We have no explanation as to why some seemingly random positions have different slopes. 
Table 2

Variation of Psychometric Function Across Eccentricity on the Horizontal Meridian for Binocular Viewing (Subject W.B.)

\begin{tabular}{|c|c|c|c|c|c|c|c|c|c|}
\hline $\begin{array}{l}\text { Eccentricity } \\
\left({ }^{\circ}\right)\end{array}$ & $\begin{array}{c}\text { No. of } \\
\text { Responses }\end{array}$ & $\begin{array}{l}\text { Weibull } \beta \\
\text { (base } e \text { ) }\end{array}$ & $\begin{array}{l}\text { Logistic } \beta \\
\quad \text { (base } e \text { ) }\end{array}$ & $\begin{array}{l}\text { Maximum } \\
\text { Slope } \\
\text { Weibull } \\
\left(p_{\mathrm{c}} / \log 10\right)\end{array}$ & $\begin{array}{l}\text { Maximum } \\
\text { Slope } \\
\text { Logistic } \\
\left(p_{\mathrm{c}} / \log 10\right)\end{array}$ & $\begin{array}{l}\text { Confidence } \\
\text { Interval } \\
\text { Logistic } \beta\end{array}$ & $\begin{array}{l}\text { Bonferroni- } \\
\text { Corrected } \\
\text { Interval } \\
\text { Logistic } \beta\end{array}$ & Significant & \\
\hline all & 17,698 & 5.43 & 9.35 & 4.14 & 4.84 & $8.83 / 9.90$ & $\mathrm{n} / \mathrm{a}$ & & \multirow{6}{*}{$\begin{array}{l}\text { Central } \\
\text { Range }\end{array}$} \\
\hline 0 & 1,518 & 4.95 & 8.77 & 3.77 & 4.54 & $7.39 / 10.30$ & $6.63 / 11.14$ & & \\
\hline 2 & 790 & 6.09 & 11.46 & 4.64 & 5.94 & $9.17 / 14.14$ & 7.91/15.61 & & \\
\hline 4 & 596 & 7.23 & 8.91 & 5.51 & 4.62 & $6.59 / 11.78$ & $5.31 / 13.36$ & & \\
\hline 6 & 847 & 4.65 & 9.00 & 3.55 & 4.66 & $7.25 / 10.91$ & $6.29 / 11.96$ & & \\
\hline 8 & 1,079 & 5.25 & 10.14 & 4.00 & 5.25 & $8.27 / 12.18$ & $7.24 / 13.30$ & & \\
\hline 10 & 1,184 & 6.17 & 11.06 & 4.70 & 5.73 & $9.22 / 13.07$ & $8.21 / 14.18$ & & \multirow{3}{*}{$\begin{array}{l}\text { Border of Blind spot: } \\
\text { Low } \beta\end{array}$} \\
\hline 12 & 1,247 & 1.08 & 3.97 & 0.82 & 2.06 & $3.25 / 4.76$ & $2.85 / 5.19$ & $*$ & \\
\hline 14 & 779 & 7.93 & 13.69 & 6.05 & 7.09 & $10.66 / 17.08$ & $8.99 / 18.94$ & & \\
\hline 16 & 672 & 6.52 & 12.28 & 4.97 & 6.36 & $9.48 / 15.41$ & $7.94 / 17.13$ & & \multirow{4}{*}{$\begin{array}{c}\text { Blind Spot } \\
\text { Border of Blind spot: } \\
\text { Low } \beta\end{array}$} \\
\hline 18 & 670 & 5.28 & 11.06 & 4.03 & 5.73 & $8.16 / 14.44$ & $6.57 / 16.30$ & & \\
\hline 20 & 985 & 4.58 & 2.91 & 3.49 & 1.51 & $2.26 / 3.69$ & $1.90 / 4.12$ & $*$ & \\
\hline 22 & 796 & 4.11 & 8.09 & 3.13 & 4.19 & $6.52 / 9.81$ & $5.66 / 10.76$ & & \\
\hline 24 & 1,104 & 5.78 & 11.62 & 4.41 & 6.02 & $9.30 / 14.30$ & $8.02 / 15.77$ & & \\
\hline 26 & 712 & 7.47 & 11.33 & 5.69 & 5.87 & $8.34 / 14.80$ & $6.70 / 16.71$ & & \\
\hline 28 & 1,000 & 6.87 & 11.93 & 5.24 & 6.18 & $9.04 / 15.13$ & $7.45 / 16.89$ & & \\
\hline 30 & 896 & 10.44 & 15.58 & 7.96 & 8.07 & $11.70 / 20.12$ & $9.57 / 22.62$ & * & Like \\
\hline 32 & 980 & 5.88 & 8.57 & 4.48 & 4.44 & $6.42 / 10.93$ & $5.24 / 12.23$ & & Central \\
\hline 34 & 854 & 3.42 & 3.64 & 2.61 & 1.89 & $2.24 / 6.08$ & $1.47 / 7.42$ & $*$ & Range \\
\hline 36 & 564 & 3.21 & 3.75 & 2.45 & 1.94 & $2.24 / 6.05$ & $1.41 / 7.32$ & $*$ & \\
\hline 38 & 350 & 4.26 & 4.52 & 3.25 & 2.34 & $2.53 / 6.86$ & $1.44 / 8.14$ & $*$ & \\
\hline 40 & 92 & 5.37 & 7.41 & 4.09 & 3.84 & $2.77 / 14.16$ & $0.22 / 17.87$ & & \\
\hline
\end{tabular}

$* p \leq 5 \%$.

Perhaps the $\chi^{2}$ approximation to the likelihood ratio, on which the significance markings are based, yields too narrow confidence intervals. In particular, the lower slope at $28^{\circ}$ seems to represent a poor fit (see Figure 9; it might stem from outliers), and the dotted line in Figure 8 at $28^{\circ}$ emphasizes the better fit of the Weibull at that eccentricity (see Treutwein \& Strasburger, 1999, for a discussion of psychometric function fitting). In any case, the fits with the Weibull function showed no significant deviation at any eccentricity (confidence intervals not listed here), so the numerical variation in the logistic's slope might have no meaning. Figure 9 shows a complete set of fits of the logistic, based on the results in Table 4 .
Figure 10 summarizes all the data for monocular viewing. Probability of being correct is plotted as a function of $\log$ relative contrast, as was done in Figure 4 for binocular viewing. The fit is about equally good as for the binocular case. There are some deviations to above-chance performance visible in the range of -0.75 to $-0.3 \log$ units below threshold, but these clearly visible points should not deter from the fact that they only constitute $2.5 \%$ of the overall data. The figure further shows that the (true) lapsing performance is not zero but somewhere between 0 and .05 , so a fit with lapsing parameter not set to a small value would give better results (Klein, 2001; Treutwein \& Strasburger, 1999; Wichmann \& Hill, 2001).

Table 3

Variation Across Eccentricity on the Horizontal Meridian, Binocular Viewing, for the Second Subject (C.H.)

\begin{tabular}{|c|c|c|c|c|c|c|c|c|}
\hline $\begin{array}{l}\text { Eccentricity } \\
\left({ }^{\circ}\right)\end{array}$ & $\begin{array}{c}\text { No. of } \\
\text { Responses }\end{array}$ & $\begin{array}{l}\text { Weibull } \beta \\
\text { (base } e \text { ) }\end{array}$ & $\begin{array}{l}\text { Logistic } \beta \\
\quad \text { (base } e \text { ) }\end{array}$ & $\begin{array}{l}\text { Maximum } \\
\text { Slope } \\
\text { Weibull } \beta \\
\left(p_{\mathrm{c}} / \log 10\right)\end{array}$ & $\begin{array}{l}\text { Maximum } \\
\text { Slope } \\
\text { Logistic } \beta \\
\left(p_{\mathrm{c}} / \log 10\right)\end{array}$ & $\begin{array}{l}\text { Confidence } \\
\text { Interval } \\
\text { Logistic } \beta\end{array}$ & $\begin{array}{l}\text { Bonferroni- } \\
\text { Corrected } \\
\text { Interval } \\
\text { Logistic } \beta\end{array}$ & Significant \\
\hline all & 16,231 & 5.53 & 9.64 & 4.22 & 4.99 & $9.04 / 10.25$ & $8.79 / 10.5$ & $\mathrm{n} / \mathrm{a}$ \\
\hline 0 & 1,420 & 2.47 & 7.32 & 1.88 & 3.79 & $5.97 / 8.79$ & $5.40 / 9.41$ & $*$ \\
\hline 2 & 1,637 & 3.80 & 8.96 & 2.90 & 4.64 & $7.32 / 10.70$ & $6.62 / 11.4$ & \\
\hline 4 & 1,239 & 5.42 & 9.48 & 4.13 & 4.91 & $7.40 / 11.80$ & $6.52 / 12.8$ & \\
\hline 6 & 1,628 & 6.26 & 11.67 & 4.77 & 6.05 & $9.51 / 14.00$ & $8.59 / 15.0$ & \\
\hline 8 & 1,597 & 6.17 & 10.70 & 4.70 & 5.54 & $8.68 / 12.90$ & $7.82 / 13.8$ & \\
\hline 10 & 1,688 & 6.05 & 9.74 & 4.61 & 5.05 & $7.89 / 11.81$ & $7.10 / 12.7$ & \\
\hline 12 & 1,728 & 5.86 & 9.73 & 4.47 & 5.04 & $7.86 / 11.83$ & $7.07 / 12.7$ & \\
\hline 14 & 1,940 & 6.03 & 10.81 & 4.60 & 5.60 & $8.68 / 13.14$ & $7.77 / 14.1$ & \\
\hline 16 & 1,529 & 5.52 & 9.69 & 4.21 & 5.02 & $7.87 / 11.69$ & $7.10 / 12.5$ & \\
\hline 18 & 1,561 & 5.49 & 9.90 & 4.19 & 5.13 & $8.06 / 11.90$ & $7.28 / 12.8$ & \\
\hline
\end{tabular}

$* p \leq 5 \%$. 
Table 4

Psychometric Function Data for Monocular Viewing on the Horizontal Meridian (Subject W.B.)

\begin{tabular}{|c|c|c|c|c|c|c|c|c|c|}
\hline $\begin{array}{c}\text { Eccentricity } \\
(\mathbf{o})\end{array}$ & $\begin{array}{c}\text { No. of } \\
\text { Responses }\end{array}$ & Threshold $\alpha$ & $\begin{array}{l}\text { Weibull } \beta \\
\text { (base } e \text { ) }\end{array}$ & $\begin{array}{c}\text { Logistic } \beta \\
\text { (base } e \text { ) }\end{array}$ & $\begin{array}{c}\text { Maximum } \\
\text { Slope } \\
\text { Weibull } \\
\left(p_{\mathrm{c}} / \log 10\right) \\
\end{array}$ & $\begin{array}{l}\text { Maximum } \\
\text { Slope } \\
\text { Logistic } \\
\left(p_{\mathrm{c}} / \log 10\right) \\
\end{array}$ & $\begin{array}{c}\text { Confidence } \\
\text { Interval } \\
\text { Logistic } \beta \\
\end{array}$ & $\begin{array}{l}\text { Bonferroni- } \\
\text { Corrected } \\
\text { Interval } \\
\text { Logistic } \beta \\
\end{array}$ & Significant \\
\hline all & 19,019 & -0.017 & 5.45 & 8.56 & 4.15 & 4.43 & $8.05 / 9.09$ & $7.81 / 9.33$ & $\mathrm{n} / \mathrm{a}$ \\
\hline 0 & 1,446 & -0.021 & 2.84 & 7.37 & 2.17 & 3.82 & $6.23 / 8.64$ & $5.70 / 9.23$ & \\
\hline 2 & 2,388 & -0.006 & 5.76 & 11.43 & 4.39 & 5.92 & $9.96 / 12.99$ & $9.28 / 13.71$ & $*$ \\
\hline 4 & 2,437 & -0.009 & 6.79 & 12.90 & 5.18 & 6.68 & $11.23 / 14.71$ & $10.46 / 15.54$ & $*$ \\
\hline 10 & 2,503 & -0.020 & 5.21 & 8.49 & 3.97 & 4.40 & $7.26 / 9.80$ & $6.69 / 10.40$ & \\
\hline 12 & 2,218 & -0.020 & 6.03 & 8.68 & 4.6 & 4.50 & $7.06 / 10.44$ & $6.31 / 11.26$ & \\
\hline 14 & 1,253 & -0.017 & 5.13 & 6.38 & 3.91 & 3.31 & $4.51 / 8.71$ & $3.64 / 9.79$ & \\
\hline 16 & 1,082 & -0.027 & 4.56 & 5.94 & 3.48 & 3.08 & $4.36 / 7.80$ & $3.63 / 8.66$ & \\
\hline 18 & 856 & -0.023 & 5.19 & 8.14 & 3.96 & 4.22 & $6.09 / 10.51$ & $5.14 / 11.61$ & \\
\hline 20 & 1,929 & -0.023 & 5.19 & 6.74 & 3.96 & 3.49 & $5.21 / 8.47$ & $4.50 / 9.27$ & \\
\hline 28 & 1,619 & -0.022 & 4.46 & 4.26 & 3.40 & 2.21 & $3.24 / 5.65$ & $2.77 / 6.29$ & $*$ \\
\hline 32 & 795 & -0.013 & 4.20 & 7.60 & 3.20 & 3.94 & $5.32 / 10.58$ & $4.26 / 11.96$ & \\
\hline 36 & 293 & -0.002 & 14.53 & 23.66 & 11.08 & 12.26 & $14.43 / 37.42$ & $10.15 / 43.80$ & $*$ \\
\hline
\end{tabular}

$* p \leq 5 \%$.

\section{Influence of the Normalization Procedure}

To study the influence of the normalization-to-threshold procedure (Equation 2) on the slope estimates, a comparison of slope estimates with versus without normalization, with otherwise fixed perceptual conditions, was conducted after the completion of the main study.

The thresholds and response data were acquired with R_Contrast on the PC (Strasburger, 1997), which is the new version of the experimental program described in the Method section, ported to MS-DOS and Windows. The data normalization and preparation for the fitting of the psychometric function was performed with new, ported, and verified versions of the analysis programs. Logistic functions were then fit with PsychoFit (Harvey, 1997), the new version of Harvey's maximum-likelihood fitting programs.

Twenty runs of, at the mean, 29.5 trials each, were run at two retinal positions (foveally and at $5^{\circ}$ ) on the horizontal meridian. One thousand one hundred eighty trials were run in total; stimulus size was $1^{\circ}$. The positions were chosen so that contrast thresholds would be significantly different between the two conditions; size was such that contrast thresholds would be close to the optimum value achievable. The observer was a young, emmetropic subject

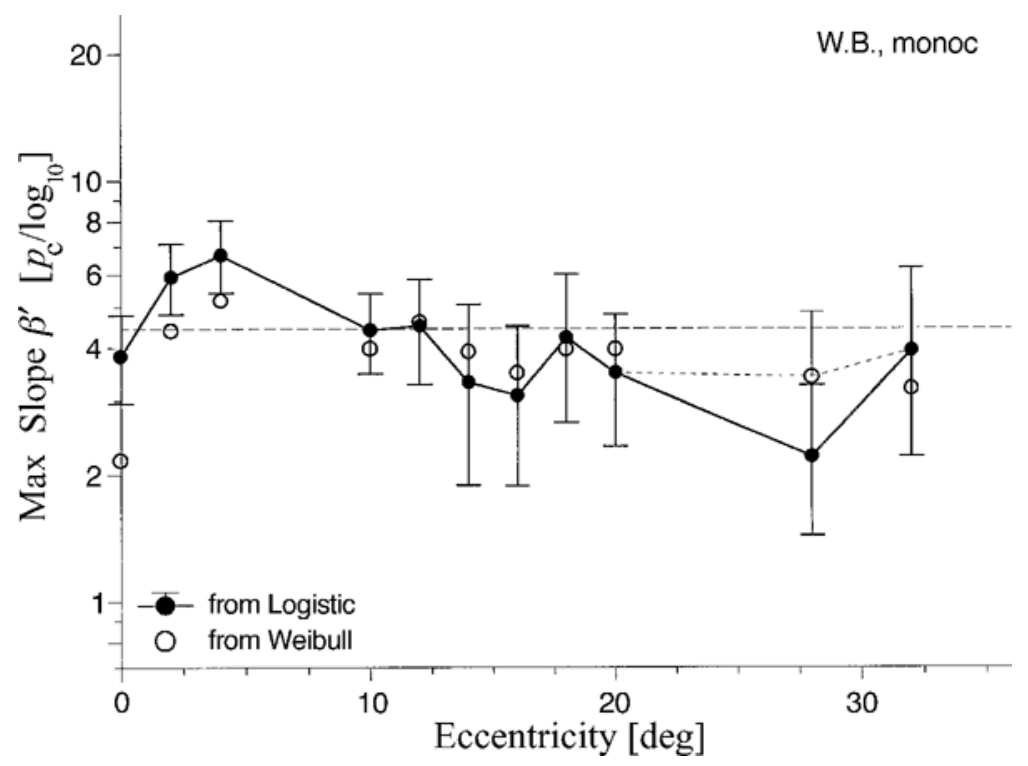

Figure 8. Slope $\beta^{\prime}$ at the point of inflection for monocular viewing on the left and right horizontal meridian, obtained from fits of the logistic (filled symbols) and Weibull (open symbols) function. Dashed line, overall fit of the logistic. Data combined for left and right eye and for left and right half-field. 


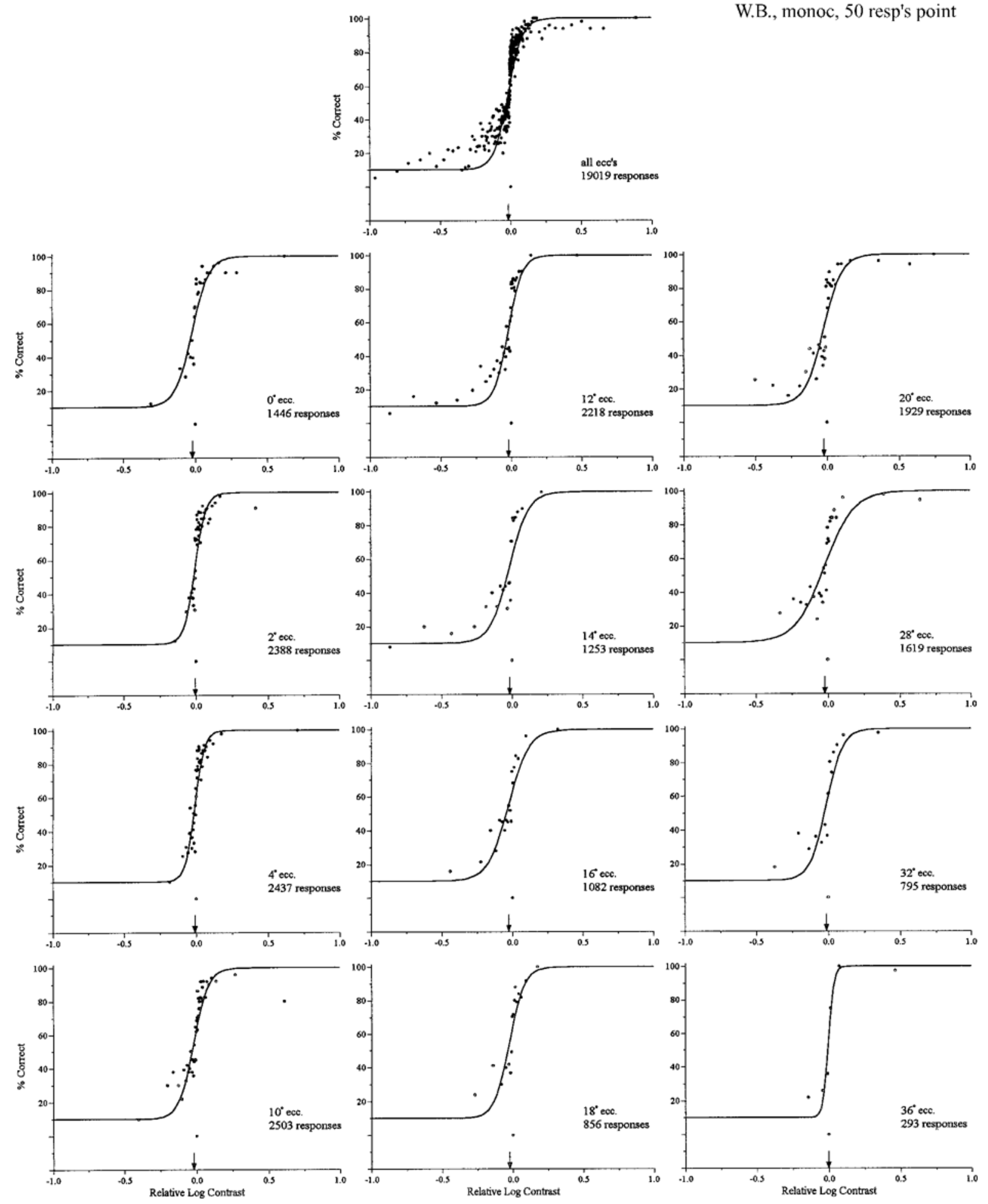

Figure 9. Psychometric functions for monocular character recognition, at various positions on the horizontal meridian. Slopes are taken from Table 4. Note a few clear response lapses at high contrast-for example, at $2^{\circ}$ and $10^{\circ}$.

who was not informed of the purpose of the experiment. Threshold estimation for each run was, as before, by maximum-likelihood of a Weibull function of fixed slope to the response data of that run. The mean, standard de- viation, and range of these thresholds was calculated. Response data were then pooled across runs and logistic functions, with threshold and slope as free parameters, fitted to those data. The results are shown in Table 5. 
Not surprisingly, thresholds are different between foveal and eccentric presentation. From the 20 threshold estimates (1 per run), the mean $\log$ threshold is $-1.96 \pm 0.03$ foveally $(1.1 \%$ Michelson contrast) and is $-1.69 \pm 0.03(2 \%$ Michelson contrast) at $5^{\circ}$ eccentricity. The threshold estimates (alpha estimates, -1.99 and -1.74 , respectively) from the (two-parameter) fits of the logistic function to the two sets of pooled data closely match these values.

The question of how the slope estimate is affected by whether or not response data are normalized to threshold will now be addressed. The 20 threshold estimates vary around the mean in a small range, 0.15 and $0.10 \log$ units (for $0^{\circ}$ and $5^{\circ}$ eccentricity, respectively; i.e., individual psychometric functions are shifted horizontally in Figure 1), and a fit to the pooled data is expected to have somewhat lower slope. Indeed, without normalization, the slope estimates (6.99 and 5.71) are lower, by about a factor of 1.5, than those obtained with normalization applied (9.42 and 9.59). The latter values are rather similar to those reported elsewhere in the paper. Note that, as can be seen from the $95 \%$ confidence intervals, the difference in slope comparing with versus without normalization is not statistically significant, even though roughly 1,200 responses may seem like a respectable set. To statistically secure the difference, more response data would be needed. Since the difference seems systematic, however, one can consider it as a tentative estimate of the influence of normalization. As a check, the data were further pooled across the two dis- similar perceptual conditions $\left(0^{\circ}\right.$ and $\left.5^{\circ}\right)$. Data are then nonstationary. Slope is down to $\beta=2.17$ that is, to one fourth of the estimate obtained with thresholds normalized, which confirms the above conclusion.

As a consequence, slope estimates obtained with long experimental runs are not strictly comparable with those obtained with short runs. Perceptual and attentional variations that show themselves in slight fluctuations of the threshold tend to decrease the slope. Since slope (or its inverse, spread) is a measure of variability, one might, of course, well consider such fluctuations as a constituent of slope. In any case, when slope estimates are compared across studies, care must be taken as to what sources of perceptual and attentional variation play a role and whether these are treated as part of the slope estimate.

\section{DISCUSSION}

Our messages are simple. (1) Except for a possible decline at high eccentricity, there are no systematic changes across the visual field in the slope (and thus the shape) of the psychometric function for character recognition as a function of stimulus contrast. (2) Slopes for character recognition (measured in short runs from adaptive procedures and with variance from threshold fluctuations removed) are around $4.8 p_{\mathrm{c}} / \log _{10} c$. (3) Slopes for recognition, found here, are generally higher than those reported by others for detection and discrimination tasks. (4) Sys-
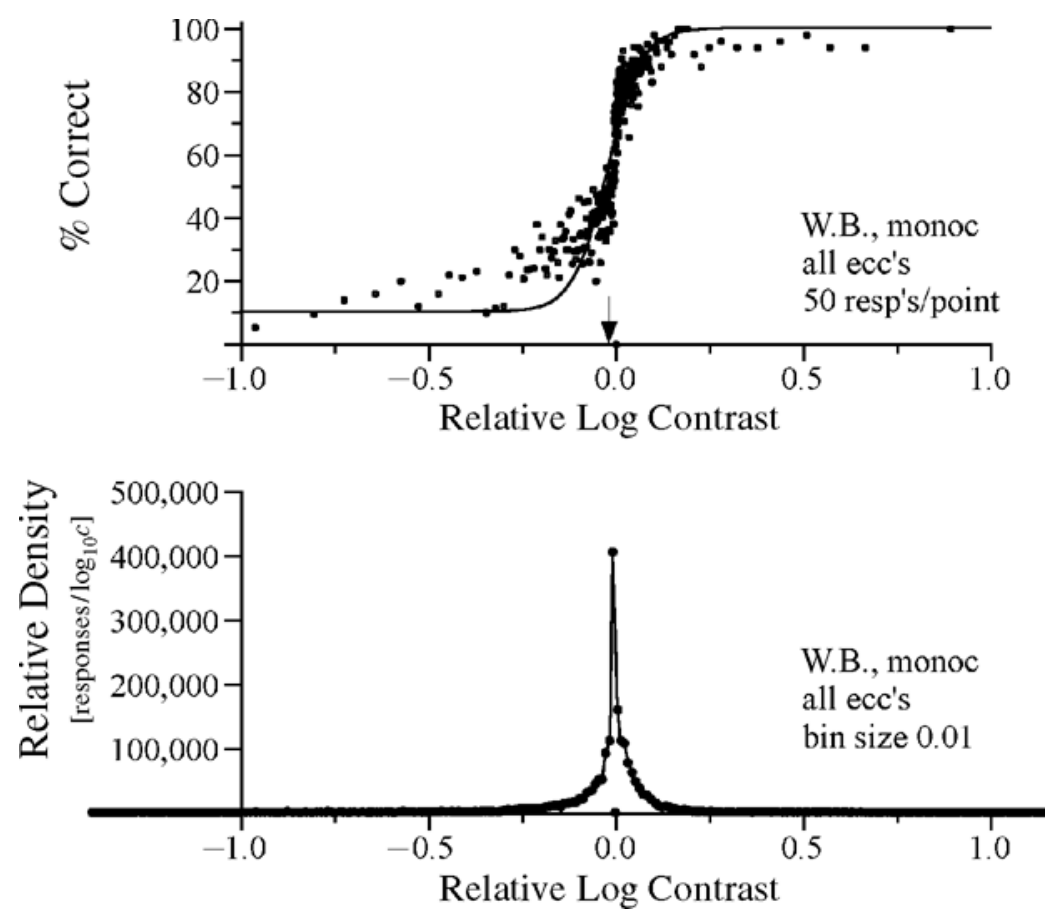

Figure 10. Summary fit for monocular viewing, Subject W.B. Under all conditions combined, there are about 19,000 responses. About nine datapoints far below threshold (representing $2.5 \%$ of the data) are peculiar outliers indicating an increase in lowcontrast performance on some occasions. 
Table 5

Comparison of Slope Estimates Obtained Without Versus With Normalization to Threshold

\begin{tabular}{|c|c|c|c|c|c|c|c|c|c|}
\hline \multirow{3}{*}{$\begin{array}{l}\text { Eccentricity } \\
(\mathbf{o})\end{array}$} & \multirow{2}{*}{\multicolumn{2}{|c|}{$\log C$}} & \multicolumn{3}{|c|}{ Without Normalization } & \multicolumn{3}{|c|}{ With Normalization } & \multirow{3}{*}{$\frac{\text { Ratio }}{b_{n} / b}$} \\
\hline & & & \multirow{2}{*}{ Alpha } & \multirow{2}{*}{\multicolumn{2}{|c|}{$\begin{array}{l}\text { Beta Confidence- } \\
\text { Interval }\end{array}$}} & \multirow[b]{2}{*}{ Alpha } & \multirow[b]{2}{*}{ Beta } & \multirow{2}{*}{$\begin{array}{c}\text { Beta Confidence- } \\
\text { Interval } \\
\end{array}$} & \\
\hline & Mean & SD/Range & & & & & & & \\
\hline 0 & -1.96 & $0.03 / 0.15$ & -1.99 & 6.99 & $4.89-9.59$ & -0.03 & 9.42 & $6.68-12.0$ & 1.35 \\
\hline 5 & -1.69 & $0.03 / 0.10$ & -1.74 & 5.71 & $3.95-7.84$ & -0.02 & 9.59 & $6.17-12.0$ & 1.68 \\
\hline 0 and 5 & -1.83 & $0.14 / 0.40$ & -1.94 & 2.17 & $1.77-2.61$ & -0.03 & 9.40 & $7.21-11.97$ & 4.33 \\
\hline
\end{tabular}

tematic changes of recognition contrast threshold with retinal locus (Strasburger \& Rentschler, 1996; Strasburger et al., 1994) are not caused by a change in the psychometric function's slope.

There are a number of points that could weaken Conclusions 1 and 2 that must be discussed before we go to Conclusions 3 and 4 . In particular, there have been reports on estimation bias that are considered first.

\section{Slope Estimation Bias}

There have been several reports that slope estimates based on data from adaptive stimulus placement strategies, unlike those from nonadaptive data, are biased toward high slopes (Kaernbach, 2001; Leek et al., 1992; Treutwein \& Strasburger, 1999), and in order to compare our results with those in other studies, the amount of bias needs to be estimated. Leek et al. were possibly the first to show that effect and to study in detail the influence of a number of parameters. They report that the bias decreases with increasing track length, with increasing number of alternatives, and with increasing true slope (holding step size constant at $2 \mathrm{~dB})$. At their shortest track length of 50 trials, at the lowest true slope ( 0.5 in units of $\left.\log _{10} d^{\prime} / \mathrm{dB}\right)$, and with a $2 \mathrm{AFC}$, there is a marked bias by a factor of 1.8 , or $80 \%$. At their highest slope of 2.0 (50 trials, 2AFC), however, the bias is rather small and around 15\% (their Figure 2). That bias is further reduced with a higher number of alternatives [estimated reduction from their Figure 1 as a factor of 1.25 for a 10AFC, scaling by number of alternatives $n$ assumed as $(1-1 / n)]$; conversely, there is an increase with a reduction to 30 trials run length, estimated as 1.1. In summary, then, from their data, at their highest slope and for 30 trials in a 10AFC, the bias would be expected as $13 \%$. In the worst case of low true slope 0.5 , probably not comparable with our case, the reduction by a $10 \mathrm{AFC}$ (instead of a 2AFC) is larger and around 1.4, so the expected bias would be $60 \%$.

In Monte Carlo simulations that we ran in our lab (Treutwein \& Strasburger, 1999), we found a prominent slope bias with data from adaptive procedures, at the mean by a factor of 1.88 (2AFC, 63 trials, spread of 10; Figure 13, p. 101), for which we had no explanation. The bias turns out to be rather similar to that found by Leek et al. (1992) in their low-slope/2AFC case and confirms their findings.

Kaernbach (2001) has provided a surprisingly obvious explanation for a slope bias from adaptive data. Since stimulus levels are not chosen at random, the random selection principle is violated, leading to a bias "in the data," not the fit. Simply put, an adaptive placement strategy will lead to responses' having, above threshold, slightly improved chances of being correct by both a selective lack of retesting after a correct response and a selective increase of retesting after an incorrect response (serial dependency). The converse is true below threshold. Taken together, this leads to a positive bias in slope estimation. ${ }^{3}$

Kaernbach (2001) has estimated the slope bias with both nonparametric and parametric maximum-likelihood estimation. The nonparametric estimate can be considered the most pure assessment of the influence of the serial dependency; from the author's Figure 1, it is slightly above $20 \%$ at a run length of 30 . The parametric estimates give higher bias, a possible reason for the difference being what Klein (2001) refers to as "shifting bias": When the threshold parameter is left free to vary in the slope estimation, fluctuations of threshold (real or apparent) will be separated from the slope estimate, thus giving higher slope estimates. Since the shifting bias is here treated separately (estimated as being around 1.5), the nonparametric estimate could be the more appropriate. As to the influence of true slope on bias, Kaernbach points out that not true slope itself, but the relation of step size to spread (inverse slope) is the governing parameter. However, unlike in up-down adaptive algorithms, the size of steps is not predefined by the experimenter in maximum-likelihood adaptive procedures, and this makes it doubtful that bias estimations from up-down procedures are directly applicable. From Figure $3 \mathrm{~A}$ (post hoc determined), step size is around $1 / 50 \mathrm{log}$ unit, or one fifth the spread, which, from Kaernbach's Figure 1, predicts a bias of 50\%. From the sample run in Figure 3A, it is also apparent, however, that there is by no means a lack of retesting after a correct response and an increase of retesting after an incorrect response (quite the opposite is true), and from that the reason for a bias is missing altogether.

Taken together, there is strong evidence that slope estimates from adaptive-procedure data are biased toward being too high. On the other hand, that bias seems to be reasonably small (13\%) for a 10AFC task of high true slope, according to Leek et al.'s (1992) simulations and around $20 \%$ in Kaernbach's (2001) estimation. It is therefore considered here that it does not alter our main conclusions.

\section{Deviations From Slope Constancy}

The fits of both the logistic and the Weibull functions result in a few significant deviations of slope from con- 
stancy. These deviations, however, occur at different positions for the two functions and show no systematic pattern. Therefore, we expect that methodical reasons, rather than characteristics of the visual system, are the possible causes. There are a number of possibilities. Weibull and logistic functions, when equated for equal maximum slope, differ in shape particularly in the range between pure guessing and subthreshold performance. Differences of slope between the two analytical functions will thus depend on data in this range, and our data show some peculiarities there that are addressed below. The validity of these slope differences thus, in part, depends on the validity of a small subset of data between a quarter and a half $\log _{10}$ unit below threshold.

The measure of reliability, on which significance markings are based, is not beyond doubt. The confidence interval for slope is based, as is often done, on the assumption of an asymptotic multivariate normality of the likelihood function - that is, that the cross-sections of the likelihood as a function of each of its parameters (in our case, slope and threshold) are asymptotically normal. How well this assumption is met depends on a number factors, including the kind of parameterization, in a way that is difficult to treat theoretically. Morgan, who has reviewed literature on the question of whether likelihood-ratio intervals are conservative or not, summarizes that a "general conclusion appears to be elusive" (Morgan, 1992, p. 65). A direct test, in our case, would be to inspect the actual shape of the likelihood functions at each eccentricity, but such an analysis needs major methodical investments that are beyond our current scope of possibilities. Another possibility is bootstrapping or jackknifing the data (Efron \& Tibshirani, 1993; Foster, 1986; Foster \& Bischof, 1997).

\section{Influence of the Stimulus Set}

Our slope estimates are for a set of characters (digits) where the members may have different contrast thresholds. Unequal thresholds will "smear out" the group psychometric function in such a way that our slope estimates constitute the lower bound for possible character sets. Alexander et al. (1997) indeed show, for foveal vision, contrast threshold differences of around $0.08 \mathrm{log}$ units between curved and angular Sloan letters for relatively large $\left(0.33^{\circ}\right)$ letter size and around $0.3 \log$ units for a letter size close to the acuity limit. Since the large majority of our digit sizes are far above the acuity limit (so that the small 0.08log-unit range would apply) and, furthermore, in Alexander et al.'s (1977) study, the mean of threshold variation over the character set is smaller than the range, I expect little change on a slope estimate if thresholds are equated across the character set.

More generally, the slope estimates found here are for a certain set of characters (digits) of a certain font, and one can ask how representative that set is. There are rather few data to answer that question. Alexander et al.'s (1997) Figure 1 shows, for foveal view, some unsystematic and rather small variation of slope across different letters-threshold differences are much more pronounced. There is thus currently no reason to assume that, once character sets are equated for threshold, psychometric function slopes will vary with character shape (except perhaps for characters with degenerate shape), but further studies are needed to answer that question.

\section{Weibull or Logistic Function?}

Which analytical function is most appropriate for describing psychophysical data, Weibull, logistic, or another? When slope is high, as is the case here, the shapes of the analytical functions in question are quite similar, and the choice seems of little practical relevance. The Weibull function is particularly popular in visual psychophysics because a similar function has lent itself to a simple modeling of psychophysical behavior through probability summation (Quick, 1974). Another choice is the cumulative normal, which for detection tasks, can be linked to signal detection theory (Green \& Swets, 1966; Legge \& Foley, 1980; Pelli, 1985; Wilson, 1980). Since the theoretical link to recognition tasks is not clear, however, there is no direct advantage to using the cumulative normal here. In their shapes, the cumulative normal and the logistic are very similar. On theoretical grounds, then, there seems to be no advantage to choosing one function over the other, and the choice can be based on which gives the better fit in a particular task. For the present data, the logistic gives slightly better fits and also a more coherent description of the data, in that it captures the blind spot border a little more accurately (the outer border at $18^{\circ}$; Figure $7 \mathrm{~A}$, Table 2), so it is the preferred one here. Note that threshold valuesthat is, the abscissa values of the point of inflection - are different between the two functions (see Figure 1)! For the mean slope of $\beta^{\prime}=5.54 p_{\mathrm{c}} / \log _{10} c$ found here (see Figure 7), this difference is $0.0149 \log _{10}$ units.

The slight threshold biases of $-0.02 \log$ units that are visible in Figures 4B and 10 are of a different origin. There are two possible causes. One, in the individual fits, the fact that the actual performance at very low contrasts was slightly higher than the expected 0.10 , led to an overestimation of threshold $\alpha$ (Swanson \& Birch, 1992). Through the normalization of response data, with $\alpha$ a little too big, threshold from the pooled data gets a negative bias. Or second, in the overall fit, the nonoptimal performance at high-contrast level with the lapsing parameter forced to zero leads directly to a negative bias. Which influence is larger cannot be decided in our data. The absolute value of the bias is rather small and has no practical meaning. Note that the bias of the point of maximum in the density plots, for binocular and monocular viewing (Figures $4 \mathrm{C}$ and 10B), which is another estimate (the small sample estimate) of the threshold bias, is much smaller, being around $0.008 \log$ units.

A few datapoints just below threshold have peculiarly higher percentage correct than was expected (see Figures 4,9 , and 10 ; between -0.75 and $-0.25 \log$ units below threshold). For binocular viewing, there are 8 datapoints 
$(8 \times 50$ responses), or $2 \%$ of the data, that intuitively seem too high. (As a check for plausibility, consider, in Figure 4, the leftmost visible point having $p_{\mathrm{c}}=.32$ : It represents 16 correct responses that had occurred between -0.96 and $-0.64 \log$ units. Assuming a binomial distribution, the point's standard deviation is $6.6 \%$-that is, such a value will occur with only $1 \%$ probability. Taken together with 7 other such points out of the total of 340 , representing 50 responses each, a deviation by chance alone is highly unlikely.) A general increase of guessing rate above.1 seems ruled out by the forced-choice design used here with stimuli randomized over the 10 digits and data summed across digits. A Weibull function, which is more rounded below threshold, will capture the peculiarly higher performance half a $\log$ unit below threshold better, and this might be an explanation for a few poor fits with the logistic. The logistic, on the other hand, gave the overall better fit. We conclude that, although the overall fit is best for the logistic function, performance just below threshold might be better described by the Weibull function.

\section{Comparison of Empirical Results}

To put the results on slope reported here into perspective, they need to be compared with those found by others. Such comparisons are summarized in Table 6. Surprisingly, no such empirical comparison seems to have been attempted before, even though there are quite a number of studies on the psychometric function for stimulus detection with contrast as the independent variable, both for foveal and eccentric vision.

The classic paper of Lamar, Hecht, Hendley, and Shlaer (1948) is based on an extensive set of data, gathered under a wide range of conditions, and the value of $2.27 p_{\mathrm{c}} / \log _{10} c$ that can be derived from their average data can serve as a reference for the case of light spot detection in foveal vision. There seems to be little difference to this value in peripheral vision (Elbel, Schaumberger, \& Lachenmayr, 1996), except that slope might be a little shallower on the vertical meridian (Chauhan \& House, 1991). For impaired vision, as in glaucoma, slope can be markedly decreased (Chauhan, Tompkins, LeBlanc, \& McCormick, 1993), and slope measurements might thus be of clinical relevance.

There are a number of studies on the psychometric function for grating detection(Blackwell, 1946, 1963; Legge, 1978; Nachmias, 1967; Sachs, Nachmias, \& Robson, 1971); the paper of Legge has been chosen as an example. From Legge's result, it seems that the psychometric function for grating detection is similar to that for unstructured stimuli, or perhaps a little shallower.

Higgins et al.'s (1996) study is the only study on the psychometric function as yet that uses characters as stimuli. The authors compare stimulus detection with discrimination-or, in their terminology, with identification. Stimuli are the specific letter pair "d" versus "b"- that is, stimuli that are mirror images of each other. Slopes are much shallower in Higgins's paradigm than in the other studies reviewed, those for detection being lower by a factor of two or three than comparable results for light spots. Slopes for discrimination are still lower, by a further factor of around two. Why Higgins's results for detection are so much lower than all the other values reported is not clear. A reason could be their use of a specific image-stabilizing technique (using rotating mirrors driven by an eye tracker), in which saccadic suppression or some other mechanism could play a different role than in conventional testing.

\section{Threshold Variation and Slope}

The slopes for digit recognition reported here are, thus, higher by a factor of close to two than all other comparable values - that is, they are remarkably high. Are there methodological reasons? One reason discussed above is a bias in the slope estimate caused by using adaptiveprocedure data, which seems to contribute perhaps $13 \%$ or a little more. A more important reason is probably that data have been effectively "stationarized" by applying the described threshold normalization to the comparably short runs of approximately 30 responses required for one threshold estimate. From validation experiments described in the Results section, that influence of normalization is estimated to explain a slope increase by a factor of about 1.5. In effect, the performance variance visible in finite slope is decomposed into threshold fluctuations lasting longer than 30 trials (removed by the normalization; 30 trials take around $60 \mathrm{sec}$ ) and remaining short-term variability. With longer experimental sessions, as in the method of constant stimuli, slopes should be shallower: There is clear evidence that thresholds, at least for a number of tasks, show rhythmic variations, possibly linked to other biorhythms. Thoss, Bartsch, and Stebel, (1998), for example, report, for photopic light spot detection at threshold $\left(5^{\circ}\right.$, $100 \mathrm{msec}$ ), highly stable rhythms during extended sessions ( $50 \mathrm{~min}$ ), with periods in the range of $0.5-15 \mathrm{~min}$ that vary between sessions and subjects (see Thoss et al., 1998, for a review; note that Thoss et al. use the term "sensitivity" to denote not inverse threshold, but proportion detected). Lotze, Roenneberg, and co-workers found daily rhythms for temporal resolution in both the visual and the auditory domains that were strongly linked to vigilance (visual double-pulse resolution, Lotze, Treutwein, \& Roenneberg, 2000; auditory fusion thresholds, Lotze, Wittmann, von Steinbüchel, Pöppel, \& Roenneberg, 1999). Amplitude of the foveal temporal-threshold variation was as large as $60 \%$. Constant-stimulus designs will incorporate these variations into ("overall") slope.

The influence of normalization will not, however, explain the differences in slope between the recognition tasks studied here and the detection tasks reported in the literature. The study of Lamar et al. (1948) used normalization similar to our's and seems representative for most of the results. We thus assume that the slope difference is due to the difference in the tasks themselves-namely, that all the other tasks considered, be they with unstructured or structured stimuli, were detection tasks. 


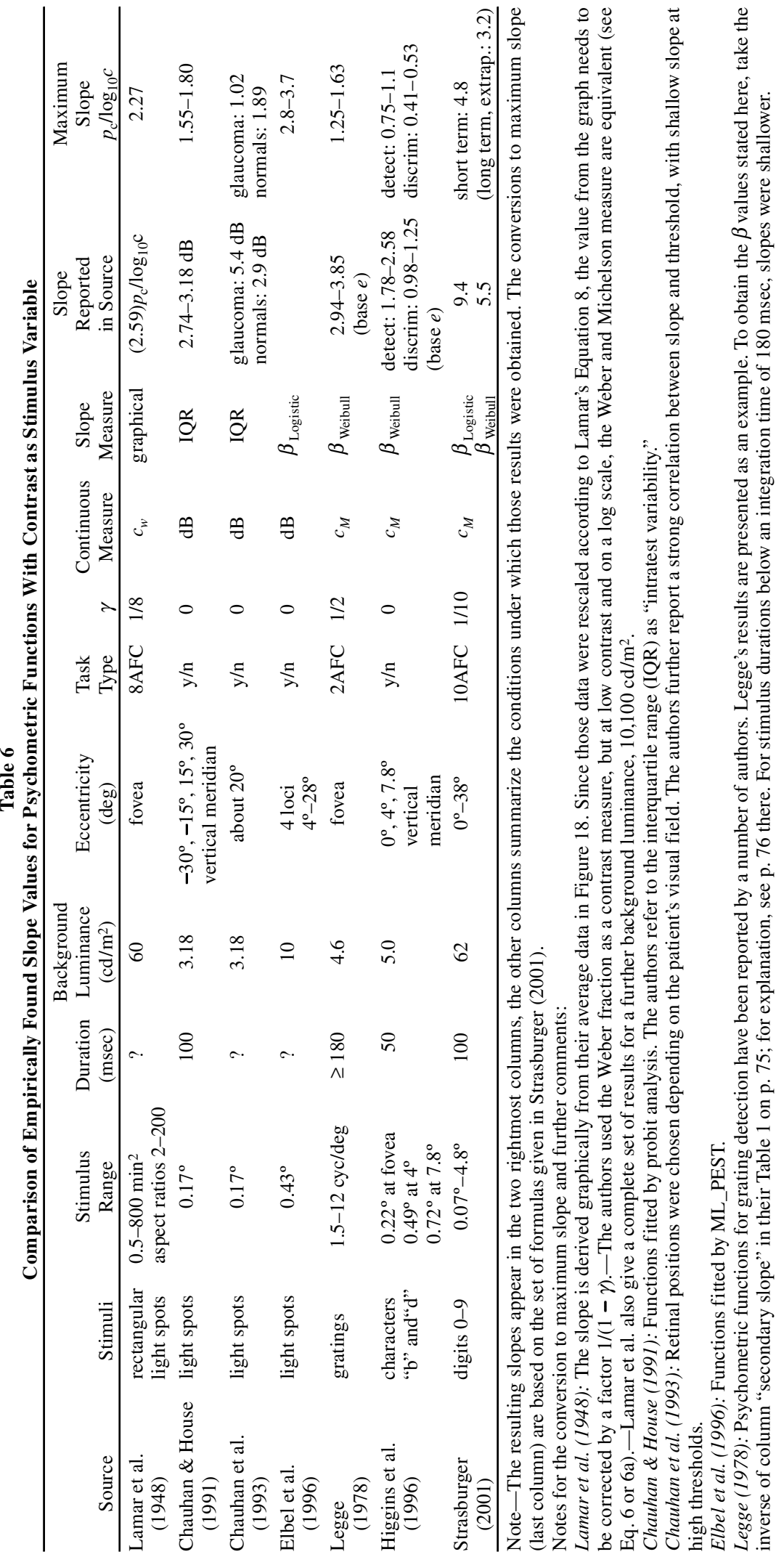




\section{Outlook}

Recognizing shapes, at arbitrary contrasts, is among the basic human perceptual abilities. It can be described, at any fixed visual field position, by a two-dimensional psychometric function $P_{\mathrm{c}}=\Psi(s, c)$ relating recognition performance (proportion correct) to pattern size and contrast, respectively. If the slopes versus the size and contrast of the function $\Psi$ are independent of each other, which needs to be shown, $\Psi$ can be described by its cross-sections along the independent axes - that is, by the one-dimensional psychometric functions of size and contrast. The latter, in turn, are for the most part well described by two variable parameters, threshold and slope, since guessing and lapsing rate can often be assumed as constant across conditions. For simple binary (i.e., black/white) target patterns, then, a good account could be given by describing the variation of just two plus two parameters over the visual field. Such a description, however, does not yet exist. Curiously lacking is, specifically, any treatment of contrast. The present report, together with previous reports on thresholds (Strasburger \& Rentschler, 1996; Strasburger et al., 1994), tries to fill this gap.

\section{CONCLUSION}

Slopes are remarkably high over a large range of conditions. Contrast thresholds are, thus, defined with high precision. There is no systematic variation of slope with retinal eccentricity. The logistic function shows a slightly better fit than the Weibull. The expected maximum slope, for the logistic function, is $\beta^{\prime}=4.8$ (increase of proportion correct per $\log _{10}$-unit increase of stimulus value); the corresponding $\beta$ values (defined on base $e$ ) are $\beta=9.4$ for the logistic and $\beta=5.5$ for the Weibull. These slope values correspond to short-term measurements (around 30 trials, or $1 \mathrm{~min}$ ) and do not include performance variations of longer duration; the latter are estimated to increase slope by a factor of about 1.5 . The function's slope is about twice that reported for a number of detection tasks. Slope far exceeds the requirement for reliable threshold measurement, so that adaptive threshold estimation procedures can be applied with high confidence. The results allow the comparison of recognition contrast thresholds across studies and should provide a firm basis for the assessment of the visual sense of form at low contrast across the visual field.

\section{REFERENCES}

Al exander, K. R., Xie, W., \& Derl acki, D. J. (1993). The effect of contrast polarity on letter identification. Vision Research, 17, 24912497.

Al exander, K. R., Xie, W., \& Derl acki, D. J. (1994). Spatialfrequency characteristics of letter identification. Journal of the Optical Society of America A, 11, 2375-2382.

Al exander, K. R., Xie, W., \& Der l acki, D. J. (1997). Visual acuity and contrast sensitivity for individual Sloan letters. Vision Research, 37, 813-819.

Bach, M., Meigen, T., \& St rasburger, H. (1997). Raster-scan cathode ray tubes for vision research: Limits of resolution in space, time and intensity, and some solutions. Spatial Vision, 10, 403-414.
Barth, E., Zet zsche, C., \& Rent schler, I. (1998). Intrinsic twodimensional features as textons. Journal of the Optical Society of America A, 15, 1723-1732.

Beck, R. W., Dieh1, L., Cl ear y, P. A., \& Group, O. N. S. (1993). The Pelli-Robson letter chart: Normative data for young adults. Clinical Vision Sciences, 8, 207-210.

Berry, G. A. (1889). Bemærkinger om retrobulbær neuritis med specielt hensyn till klar hedssansens tilstand vid denne ledelse. Royal London Ophthalmic Hospital Reports and Journal of Ophthalmic Medicine \& Surgery, 12.

Bl ackwel 1, H. R. (1946). Contrast thresholds of the human eye. Journal of the Optical Society of America, 36, 624-643.

Bl ackwel 1, H. R. (1963). Neural theories of simple visual discrimination. Journal of the Optical Society of America, 53, 129-160.

Bl omma er t, F. J., \& Timmer s, H. (1987). Letter recognition at low contrast levels: Effects of letter size. Perception, 16, 421-432.

Brown, V. A., Dor an, R. M. L., \& Woodhou se, J. M. (1987). The use of computerized contrast sensitivity, Arden gratings and low contrast letter charts in the assessment of amblyopia. Ophthalmology \& Physiological Optics, 7, 43-51.

Campbel1, F. W., \& Gr een, D. M. (1965). Optical and retinal factors affecting visual resolution. Journal of Physiology, 181, 576-593.

Chau han, B. C., \& Hou se, P. H. (1991). Intratest variability in conventional and high-pass resolution perimetry. Ophthalmology, 98, 79-83.

Chau han, B. C., Tompkins, J. D., LeBl anc, R. P., \& McCor mick, T. A. (1993). Characteristics of frequency-of-seeing curves in normal subjects, patients with suspected glaucoma, and patients with glaucoma. Investigative Ophthalmology \& Visual Science, 34, 3534-3540.

Coll et t, D. (1991). Modelling binary data. London: Chapman and Hall.

Drasdo, N. (1991). Neural substrates and threshold gradients of peripheral vision. In J. J. Kulikowski, V. Walsh, \& I. J. Murray (Eds.), Limits of vision (pp. 250-264). London: Macmillan.

Ef ron, B., \& Tibshir ani, R. J. (1993). An introduction to the bootstrap. New York: Chapman and Hall.

El bel, G.-K., Sch au mber ger, M. M., \& Lach en mayr, B. J. (1996). Psychometric functions and frequency-of-seeing curves for lightsense perimetry. Unpublished manuscript.

Finney, D. J. (1971). Probit analysis. Cambridge: Cambridge University Press.

Fost er , D. H. (1986). Estimating the variance of a critical stimulus level from sensory performance data. Biological Cybernetics, 53, 189-194.

Fost er , D. H., \& Bisch of, W. F. (1997). Bootstrap estimates of the statistical accuracy of thresholds from psychometric functions. Spatial Vision, 11, 135-139.

Fr ance, T. D., \& Fr ance, L. W. (1988). Low contrast visual acuity cards in pediatric ophthalmology. Albrecht von Graefes Archiv für klinische und experimentelle Ophthalmologie, 226, 158-160.

Gr ah am, N., \& Robson, J. G. (1987). Summation of very close spatial frequencies: The importance of spatial probability summation. $\mathrm{Vi}$ sion Research, 27, 1997-2007.

Green, D. M. (1995). Maximum-likelihood procedures and the inattentive observer. Journal of the Acoustical Society of America, 97, 3749-3760.

Green, D. M., \& Swet s, J. A. (1966). Signaldetection theory and psychophysics. New York: Wiley. Reprint edition (1988) by Peninsula Publishing, Los Altos.

Har vey, L. O., Jr . (1986). Efficient estimation of sensory thresholds. Behavior Research Methods, Instruments, \& Computers, 18, 623632 .

Har vey, L. O., Jr . (1997). Efficient estimation of sensory thresholds with ML-PEST. Spatial Vision, 11, 121-128.

Harvey, L. O., Jr ., \& Gerva is, M. J. (1978). Visual texture perception and Fourier analysis. Perception \& Psychophysics, 24, 534-542.

Hays, W. L. (1981). Statistics. New York: Holt, Rinehart and Winston.

Her se, P. R., \& Bedel 1, H. E. (1989). Contrast sensitivity for letter and grating targets under various stimulus conditions. Optometry \& Vision Science, 66, 774-781.

Higgins, K. E., Ar dit i, A., \& Knoblauch, K. (1996). Detection and identification of mirror-image letter pairs in central and peripheral vision. Vision Research, 36, 331-337.

Kaer nbach, C. (2001). Slope bias of psychometric functions derived from adaptive data. Perception \& Psychophysics, 63, 1389-1398. 
King-Smit h, P. E., \& Rose, D. (1997). Principles of an adaptive method for measuring the slope of the psychometric function. Vision Research, 37, 1595-1604.

$\mathrm{Kl}$ ein, S. P. (2001). Measuring, estimating and understanding the psychometric function: A commentary. Perception \& Psychophysics, 63, 1421-1455.

Kont sevich, L. L., \& Tyl er, C. W. (1999). Bayesian adaptive estimation of psychometric slope and threshold. Vision Research, 39, 2729 2737.

Lamar, E. S., Hech t, S., Hendl ey, C. D., \& Shl aer, S. (1948). Size, shape, and contrast in detection of targets by daylight vision: II. Frequency of seeing and the quantum theory of cone vision. Journal of the Optical Society of America, 38, 741-755.

Leek, M. R., Hanna, T. E., \& Mar sha11 ,L. (1992). Estimation of psychometric functions from adaptive tracking procedures. Perception \& Psychophysics, 51, 247-256.

Legge, G. E. (1978). Sustained and transient mechanisms in human vision: Temporal and spatial properties. Vision Research, 18, 69-81.

Legge, G. E., \& Fol ey, J. M. (1980). Contrast masking in human vision. Journal of the Optical Society of America, 70, 1458-1471.

Legge, G. E., Rubin, G. W., \& Luebker, A. (1987). Psychophysics of reading: V. The role of contrast in normal vision. Vision Research, 27, 1165-1177.

Lel kens, A. M., \& Opponeer, P. M. (1983). The frequency of seeing square wave density modulations in random dot patterns. Biological Cybernetics, 48, 165-174.

Levi, D. M., \& Kl ein, S. A. (1985). Vernier acuity, crowding and amblyopia. Vision Research, 25, 979-991.

Link, S. W. (1992). The wave theory of difference and similarity. Hillsdale, NJ: Erlbaum.

Lot ze, M., Tr eut wein, B., \& Roenneber g, T. (2000). Daily rhythm of vigilance assessed by temporal resolution of the visual system. Vision Research, 40, 3467-3473.

Lot ze, M., Wit t mann, M., von St ein büchel , N., Pöppel, E., \& Roenneber g, T. (1999). Daily rhythm of temporal resolution in the auditory system. Cortex, 35, 89-100.

Mackeben, M. (1999). Sustained focal attention and peripheral letter recognition. Spatial Vision, 12, 51-72.

Mayer, M. J., \& Tyl er, C. W. (1986). Invariance of the slope of the psychometric function with spatial summation. Journal of the Optical Society of America A, 3, 1166-1172.

Morgan, B. J. T. (1992). Analysis of quantal response data. London: Chapman and Hall.

Nach mias, J. (1967). The effect of exposure duration on visual contrast sensitivity with square-wave gratings. Journal of the Optical Society of America, 57, 421-427.

Nach mias, J. (1981). On the psychometric function for contrast detection. Vision Research, 21, 215-223.

Pel 1 i, D. G. (1985). Uncertainty explains many aspects of visual contrast detection and discrimination. Journal of the Optical Society of America A, 2, 1508-1532.

Pell i, D. G. (1987). On the relation between summation and facilitation. Vision Research, 27, 119-123.

Pell 1 i, D. G., Robson, J. G., \& Wil kin s, A. J. (1988). The design of a new letter chart for measuring contrast sensitivity. Clinical Vision Sciences, 2, 187-199.

Qu ick, R. F. A. (1974). A vector magnitude model of contrast detection. Kybernetik, 16, 65-67.

Rabin, J. (1995). Small letter contrast sensitivity: An alternative measure of visual resolution for aviation candidates. Aviation Space \& Environmental Medicine, 66, 56-58.

Rabin, J., \& Wicks, J. (1996). Measuring resolution in the contrast domain: The small letter contrast test. Optometry \& Visual Science, 73, 398-403.

Regan, D. (1988a). Low-contrast letter charts and sinewave grating tests in ophthalmological and neurological disorders. Clinical Vision Sciences, 2, 235-250.

Regan, D. (1988b). Low-contrast visual acuity test for pediatric use. Canadian Journal of Ophthalmology, 23, 224-227.

Regan, D., \& Neima, D. (1983a). Low-contrast letter charts as a test of visual function. Ophthalmology, 90, 1192-1200.
Regan, D., \& Neima, D. (1983b). Low-contrast letter charts in early diabetic retinopathy, ocular hypertension, glaucoma and Parkinson's disease. British Journal of Ophthalmology, 68, 885-889.

Rovamo, J., Vir su, V., \& Näsän en, R. (1978). Cortical magnification factor predicts the photopic contrast sensitivity of peripheral vision. $\mathrm{Na}$ ture, 271, 54-56.

Sach s, M. B., Nach mias, J., \& Robson, J. G. (1971). Spatial frequency channels in human vision. Journal of the Optical Society of America, 61, 1176-1186

Sol omon, J. A., \& Pel1 i, D. G. (1994). The visual filter mediating letter identification. Nature, 369, 395-397.

St $r$ a sbu r ger, H. (1996). Formerkennung im zentralen und peripheren Gesichtsfeld. Habilitationsschrift [Recognition of form in central and peripheral vision: Habilitation thesis]. München: Medizinische Fakultät der Universität München.

St r a sburger, H. (1997). R_Contrast: Rapid measurement of recognition contrast thresholds. Spatial Vision, 10, 495-498.

St $r$ a sbur ger, H. (2001). Converting between measures of slope of the psychometric function. Perception \& Psychophysics, 63, 1348-1355.

St $r$ a sbur ger, H. (2002). Indirektes Sehen: Formerkennung im zentralen und peripheren Gesichtsfeld [Indirect vision: Recognition of form in central and peripheral vision]. Göttingen: Hogrefe.

St $r$ a sbu r ger, H., Har vey, L. O., Jr ., \& Rent sch 1 er , I. (1991). Contrast thresholds for identification of numeric characters in direct and eccentric view. Perception \& Psychophysics, 49, 495-508.

St r a sburger, H., \& Rent schl er, I. (1996). Contrast-dependent dissociation of visual recognition and detection fields. European Journal of Neuroscience, $\mathbf{8}, 1787-1791$.

St r a sbur ger, H., Rent schl er, I., \& Har vey, L. O., Jr . (1994). Cortical magnification theory fails to predict visual recognition. European Journal of Neuroscience, $\mathbf{6}, 1583-1588$.

Swanson, W. H., \& Birch, E. E. (1992). Extracting thresholds from noisy psychophysical data. Perception \& Psychophysics, 51, 409-422.

Th oma s, J. P. (1987). Effect of eccentricity on the relationship between detection and identification. Journal of the Optical Society of America A, 4, 1599-1605.

Thoss, F., Bart sch, B., \& St ebel, J. (1998). Analysis of oscillations of the visual sensitivity. Vision Research, 38, 139-142.

Tr eut wein, B. (1995). Minireview: Adaptive psychophysical procedures. Vision Research, 35, 2503-2522.

Treut wein, B., \& St r asburger, H. (1999). Fitting the psychometric function. Perception \& Psychophysics, 61, 87-106.

Vir su, V., Näsän en, R., \& Osmoviit a, K. (1987). Cortical magnification and peripheral vision. Journal of the Optical Society of America A, 4, $1568-1578$

Wat a nabe, S. (1985). Pattern recognition: Human and mechanical. New York: Wiley.

Weymout h, F. W. (1958). Visual sensory units and the minimal angle of resolution. American Journal of Ophthalmology, 46, 102-113.

Wichmann, F. A., \& Hil 1, N. J. (2001). The psychometric function: I. Fitting, sampling, and goodness of fit. Perception \& Psychophysics, 63, 1293-1313.

Wil son, H. R. (1980). A transducer function for threshold and suprathreshold human vision. Biological Cybernetics, 38, 171-178.

Zet zsche, C., \& Bart h, E. (1990). Fundamental limits of linear filters in the visual processing of two-dimensional signals. Vision Research, $\mathbf{3 0}$, 1111-1117.

\section{NOTES}

1. Triggered by Stanley Klein's (2001) comments (which are based on Wichmann \& Hill, 2001a), I checked the dependency of the slope estimates presented here on the value of the lapse parameter, $\lambda$ (Subject W.B., overall fit). Unexpectedly, there was indeed a dependency on $\lambda$, as predicted by Wichmann and Hill (2001a), and quite strong indeed even though the differences between the values of lambda seem rather small. The value used in the paper $(0.001=0.01 \%)$ is marked by an asteriskwith more realistic, larger values of $\lambda$, the slope estimates are even higher than they already are, such that the conclusion of slopes being particularly high with the recognition task presented here is amplified. 
Table N1

Dependency on the Slope Parameter on the Lapse Parameter

\begin{tabular}{lcc}
\hline Lambda & Beta Logistic & Beta Weibull \\
\hline 0.05 & 12.2016 & 8.0990 \\
0.01 & 10.4538 & 6.1171 \\
0.001 & 9.8135 & 5.5211 \\
$0.0001^{*}$ & 9.3513 & 5.4276 \\
0.00001 & 8.7702 & 5.4007 \\
0.000001 & 8.4166 & 5.3326 \\
\hline
\end{tabular}

2. The current version of MLPFIT has been renamed PsychoFit and is available from Harvey's homepage, http://psych.colorado.edu/ lharvey /home.html. Please do not confuse maximum-likelihood psychometric function fitting (PsychoFit) with maximum-likelihood adaptive procedures (like ML-PEST, QUEST, etc.).
3. Stephen Link (personal communication) proposed a further reason why with adaptive procedures, slope could be higher than with the method of constant stimuli. The argument is-intuitively-as follows: Difficult decisions, like those near threshold, take longer but are performed with higher accuracy, a fact that can be accounted for in a formal theory (wave theory; Link, 1992). Adaptive procedures, in comparison with the method of constant stimuli, pose a higher proportion of difficult sensory decisions (at threshold) which will lead to higher percent correct just above threshold. This in turn leads to higher slope. It would be interesting to see a direct test of that proposition. Note that this account, if it holds up in a direct test, would imply that the high slopes with adaptive data, in comparison with constant stimuli or spread-out stimuli as in Kontsevich and Tyler's (1999) approach, reflect more faithfully what the sensory system can do if it needs to.

(Manuscript received September 23, 1998; revision accepted for publication March 4, 2001.) 